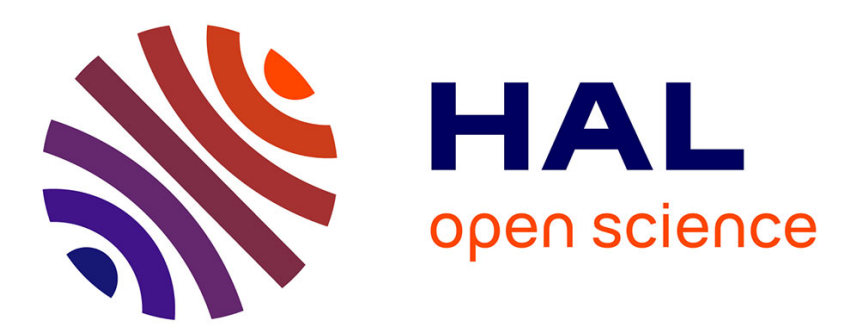

\title{
Simulation of nonlinear transient elastography: finite element model for the propagation of shear waves in homogeneous soft tissues
}

\author{
W. Ye, A. Bel-Brunon, S. Catheline, Alain Combescure, M. Rochette
}

\section{To cite this version:}

W. Ye, A. Bel-Brunon, S. Catheline, Alain Combescure, M. Rochette. Simulation of nonlinear transient elastography: finite element model for the propagation of shear waves in homogeneous soft tissues. International Journal for Numerical Methods in Biomedical Engineering, 2018, 34 (1), 10.1002/cnm.2901 . hal-01864359

\section{HAL Id: hal-01864359 \\ https://hal.science/hal-01864359}

Submitted on 29 Aug 2018

HAL is a multi-disciplinary open access archive for the deposit and dissemination of scientific research documents, whether they are published or not. The documents may come from teaching and research institutions in France or abroad, or from public or private research centers.
L'archive ouverte pluridisciplinaire HAL, est destinée au dépôt et à la diffusion de documents scientifiques de niveau recherche, publiés ou non, émanant des établissements d'enseignement et de recherche français ou étrangers, des laboratoires publics ou privés. 


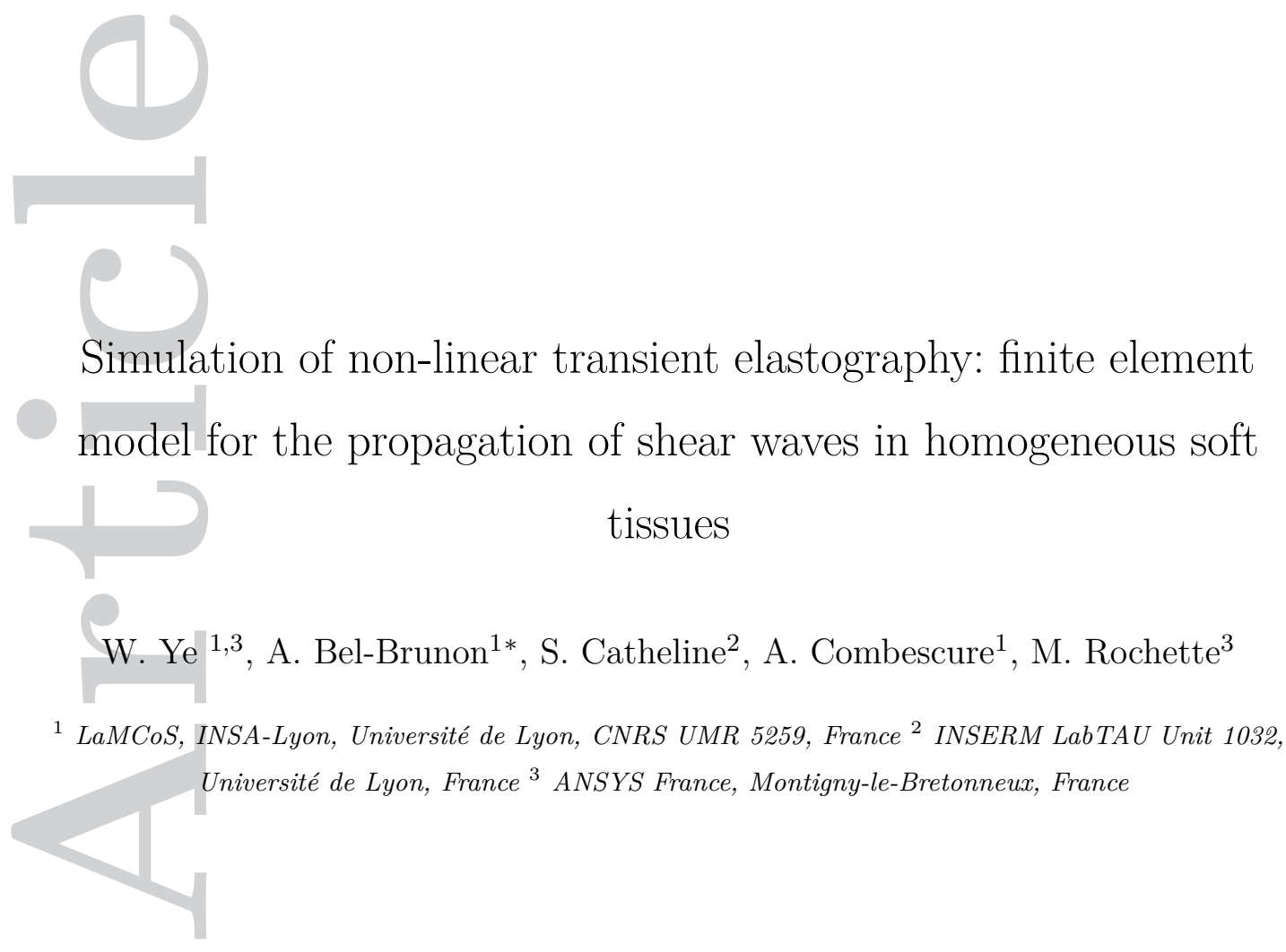

SUMMARY

In this study, visco-hyperelastic Landau's model which is widely used in acoustical physic field is introduced into a finite element formulation. It is designed to model the non-linear behaviour of finite amplitude shear waves in soft solids, typically, in biological tissues. This law is employed in finite element models based on elastography experiments reported in [1], the simulations results show a good agreement with the experimental study : it is observed in both that a plane shear wave generates only odd harmonics and a nonplane wave generates both odd and even harmonics in the spectral domain. In the second part, a parametric study is carried out to analyze the influence of different factors on the generation of odd harmonics of plane wave. A quantitative relation is fitted between the odd harmonic amplitudes and the non-linear elastic parameter of Landau's model, which provides a practical guideline to identify the nonlinearity of homogeneous tissues using elastography experiment.

Received ...

KEY WORDS: non-linear shear wave; elastography; hyperelasticity; finite element

*Correspondence to: aline.bel-brunon@insa-lyon.fr

This article has been accepted for publication and undergone full peer review but has not been through the copyediting, typesetting, pagination and proofreading process, which may lead to differences between this version and the Version of Record. Please cite this article as Int. J. Numer. Meth. Biomed. Engng., e02901. doi: 10.1002/cnm.2901 


\section{INTRODUCTION}

Transient elastography is a medical imaging technique which characterizes the elastic properties of biological tissues in vivo by observing shear wave propagation. These mechanical properties provide useful clinical information for diagnosis process.

Generally, the transient elastography technique can be described as follows: firstly, a small and low frequency shear wave is generated by a mechanical vibrator [2] or by the acoustic radiation force [3]. Then the dynamic response of the tissue is measured via ultrasonic speckle-tracking methods and the shear wave speed is computed. Assuming that the soft tissue is homogeneous, isotropic and linear elastic, the shear modulus $\mu$ can be estimated by:

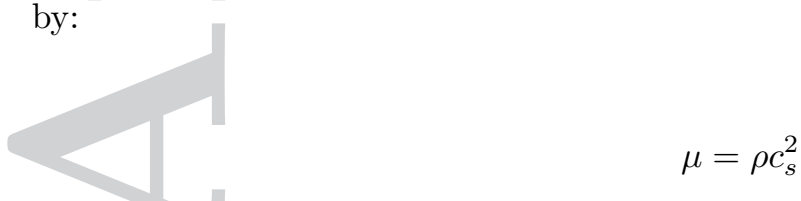

where $\rho$ is the mass density (constant) and $c_{s}$ is the shear wave speed. Although the measurement could be affected by multiple factors, such as boundary conditions, frequency of excitation or internal pressure in organs, this technique has been clinically proven to be able to evaluate many diseases, such as cancers and liver fibrosis. For example, elastography often takes advantage on inverse techniques $[4,5]$ to detect tumours, which is due to the fact that tumours are often stiffer than the surrounding healthy tissues, so the shear wave speeds are also different.

Lately, more efforts have been made to determine the non-linear properties of biological soft tissues. For example, Catheline et al. [6,1] measured the non-linear behaviour of shear waves at a high level of amplitude. Gennisson et al.[7, 8] used the so called acousto-elastic effect to determine the non-linear elastic parameters: it corresponds to measuring smallamplitude (linear) waves in pre-deformed states. These experiments were carried out on artificial gel phantoms. More recently, Jiang et al. $[9,10]$ developed their experiments for ex vivo and in vivo biological tissues. At the same time, theoretical work is necessary to support experimental results. In the work of $[11,12,13,14]$, analytical models are established but the solutions can be achieved only under simple cases.

The Finite Element Method (FEM) provides the approximate solution of non-linear partial differential equations on complex domains, and thus, can be used to analyze non-linear shear wave propagation. In the literature on the simulation of transient elastography, Chen et al. $[15,16]$ investigated different effects on the shear wave speed, such as boundary conditions and excitation frequency, through simple FEM models. To take into account 
the heterogeneity (tumours in healthy tissues), 2D [17] and 3D [18] finite element models have been proposed. In addition, different inverse approaches based on FEM [19, 20] have been proposed to reconstruct the stiffness map of biological tissues by observing shear wave propations. To our knowledge, most of the above-mentioned models use linear elastic materials (Hooke's law) with or without viscosity, but very few consider material nonlinearity [10].

Our objective in this work is to accurately model the non-linear shear wave propagation in soft solids by FEM. FEM models may indeed be valuable to find a simple method to identify non-linear parameters of tissues. To achieve this, a reliable material model is necessary: visco-hyperelastic Landau's model in the case of incompressibility is a potential choice because it has been largely used in the above-mentioned experimental and theoretical work $[6,1,7,8,11,12,13,14]$. However, to our knowledge, the numerical study of this model is rare. Most recently, Giammarinaro et al. [21] reported the numerical analysis of shock waves in brain with Landau's model. However, the work was carried out by Finite Difference method (FDM), a FEM model is still lacking. Moreover, non-linear wave propagation occurs widely in many systems. In addition to elastography, it has been investigated in the field of medical ultrasound [22], geophysics [23], vibration [24], seismology [25] and so on. In either field, FEM is considered as a generalized tool, and the development of material models is always essential.

After this introduction, the paper is structured as follow. Section 2 presents non-linear models of nearly incompressible soft solids. The weakly non-linear elastic theory of the forthorder Landau's law is firstly developed in the finite element formulation, simple numerical tests are carried out to compare its behaviour with common hyperelastic laws. Furthermore, in order to model non-linear shear waves in soft solids, visco-elasticity is considered by a Voigt model.

In section 3, the FE simulations are carried out to model the non-linear shear wave propagation in soft solids. The models are based on the experimental work of Jacob et al. reported in [1], in which both plane and nonplane shear wave experiments are carried out. To our knowledge, this is the first time that non-linear shear waves in nearly incompressible Landau's solid is analysed by FEM.

In section 4, a parametric analysis of the FE model of plane shear waves propagation is performed for the following parameters: frequency and amplitude of excitation, viscosity and nonlinearity of Landau's material. These parameters contain all the factors that may affect the non-linear behaviour of plane waves in homogeneous solids. The numerical results are compared with the analytical solution, and a practical method is proposed to identify 
the non-linear parameter of a homogeneous tissue. Finally, a conclusion is given at the end of the paper.

\section{MATERIAL MODELING}

\subsection{Hyperelasticity}

In biomechanics, choosing the accurate material model is a key requirement to get highfidelity numerical simulations. As biological materials are subjected to large deformation, they are generally modelled by hyperelastic models, where the constitutive relation is described by a strain energy function $W$. However, the fields of solid mechanics and non-linear acoustic refer to different non-linear constitutive laws and the connection and difference between these laws are still not clear.

In this section, we describe briefly some widespread hyperelastic laws in non-linear solid mechanics as well as Landau's law, which is itself generally employed in non-linear acoustic. Then, the implementation of Landau's law is presented in details. A comparison between these models is given using FE simulations.

\subsubsection{Continuum mechanics model: Rivlin model}

Let $\mathbf{F}$ denote the deformation gradient tenor, $\mathbf{C}=\mathbf{F}^{T} \mathbf{F}$ the right Cauchy-Green strain, and $J=\operatorname{det} \mathbf{F}$ the Jacobian which represents the volume change. For an isotropic hyperelastic material, the strain energy function $W$ is expressed by the invariants of tensor $\mathbf{C}$ [26]. They are defined by

$$
I=\operatorname{tr}(\mathbf{C}), \quad I I=\frac{1}{2}\left[I^{2}-\operatorname{tr}\left(\mathbf{C}^{2}\right)\right], \quad I I I=\operatorname{det} \mathbf{C}=J^{2}
$$

Many hyperelastic models have been proposed for materials which stress-strain relationship can be defined as non-linear elastic and independent of the strain rate. In this study, Rivlin model [27] is taken into consideration because it has a similar behaviour to Landau's law presented in the following. Besides, Rivlin model has been widely used to model rubber-like materials. Following the condition of incompressibility, Rivlin hyperelastic potential is:

$$
W_{\text {Rivlin }}=c_{10}(I-3)+c_{01}(I I-3)+c_{20}(I-3)^{2}
$$


where $c_{10}, c_{01}$ and $c_{20}$ are the three material constants. By setting $c_{20}=0$, we obtain the Mooney-Rivlin model; by setting both $c_{01}$ and $c_{20}$ to 0 , we obtain the neo-Hookean model, which is the simplest hyperelastic model. These are special cases of the Rivlin model.

In a recent study, Mangan et al. [28] have shown that both neo-Hookean and MooneyRivlin models lead to an fully linear response in shear deformation. However, soft solids exhibit non-linearity also in shear, such as silicone rubber [29] and agar-gelatine mixed gel [1] which is used to mimic the behaviour of biological tissues in medical acoustics studies. Therefore, using these two models leads to unrealistic numerical simulations for shear dominated responses, this point will be detailed in Section 2.2.2.

\subsubsection{Non-linear acoustic model: Landau's law}

In seismology and physic acoustics, the weakly non-linear theory [13] developed by Landau and Lifshitz [30] is widely used. Hence, the strain energy function $W$ is written in terms of Green strain tensor $\mathbf{E}=\frac{1}{2}(\mathbf{C}-\mathbf{1})$, and $\mathbf{1}$ is the second-order identity tensor. Assuming that $W$ can be approximated by a series expansion, at the fourth order, we have:

$$
W=\frac{\lambda}{2} I_{1}^{2}+\mu I_{2}+\frac{A}{3} I_{3}+B I_{1} I_{2}+\frac{C}{3} I_{1}^{3}+E I_{1} I_{3}+F I_{1}^{2} I_{2}+G I_{2}^{2}+H I_{1}^{4}
$$

with $I_{k}=\operatorname{tr}\left(\mathbf{E}^{k}\right)$ for $k=1,2,3$.

It can be seen that the model contains two second-order elastic moduli $\lambda$ and $\mu$ (Lamé coefficients), three third-order elastic constants $A, B$ and $C$, and four forth-order elastic constants $E, F, G$ and $H$. Notice that at the second order of approximation (i.e. keep only the second-order elastic moduli $\lambda$ and $\mu$ ), Landau's model is the same as the so-called St. Venant-Kirchhoff material in continuum mechanics [31].

For soft materials, Catheline et al. [8] reported the measurement of elastic constants of mixed gels up to the third order (neglecting the terms involving $E, F, G$ and $H$ ). The values of $\mu$ and $A$ are in the order of $k P a$, whereas the values of $\lambda, B$ and $C$ are in the order of $G P a$; this huge difference is attributed to the high incompressibility. Following this measurement, Hamilton et al. [32] developed the uncoupled form of the strain energy function, in which the volumetric and deviatoric components are separated:

$$
\begin{aligned}
W & =W^{\text {dev }}+W^{v o l} \\
\text { with } & W^{\text {dev }}=\mu I_{2}+\frac{A}{3} I_{3}+D I_{2}^{2}
\end{aligned}
$$


where $W^{\text {dev }}$ indicates the strain energy related to deviatoric (shear) strain, so $\mu, A$ and $D$ represent the second, third, and fourth order shear moduli. They all have the same order of magnitude $(k P a)$ in soft solids, and they play a key role in non-linear shear wave propagation.

The volumetric strain energy is not given in [32]. However, this is trivial because it plays the role of penalty function in FEM to enforce the incompressibility constraint. Therefore, we take the simplest expression as:

$$
W^{v o l}=\frac{1}{2} K(J-1)^{2}
$$

with $K$ the bulk modulus which is several orders larger than the shear modulus, generally, in the order of $G P a$.

This model is employed in a number of works to study the behaviour of soft solids in both theoretical works $[11,14,13]$ and experimental works $[9,10]$. However, there is still no numerical simulation in FEM including Landau's model, this deficiency is addressed in this paper.

\subsubsection{Implementation}

For a hyperelastic model, the second Piola-Kirchhoff (PK-2) stress $\mathbf{S}$ is derived from the strain energy, so that

$$
\mathbf{S}=\frac{\partial W}{\partial \mathbf{E}}
$$

With the following relations:

$$
\frac{\partial J}{\partial \mathbf{C}}=\frac{1}{2} J \mathbf{C}^{-1}, \frac{\partial \operatorname{tr}(\mathbf{E})}{\partial \mathbf{E}}=\mathbf{1}
$$

and making use of the chain rule in Eq.5 leads to:

$$
\begin{aligned}
\mathbf{S} & =2 \frac{\partial W^{v o l}}{\partial \mathbf{C}}+\frac{\partial W^{d e v}}{\partial \mathbf{E}} \\
& =p J \mathbf{C}^{-1}+2 \mu \mathbf{E}+A \mathbf{E}^{2}+4 D \operatorname{tr}\left(\mathbf{E}^{2}\right) \mathbf{E}
\end{aligned}
$$

where $p$ indicates the hydrostatic pressure, and is obtained by $p=K(J-1)$.

Some FEM codes are based on the current configuration, so the Cauchy stress tensor $\boldsymbol{\sigma}$ is necessary. It can be obtained by the transformation relation as: 


$$
\boldsymbol{\sigma}=\frac{1}{J} \mathbf{F S F}^{T}
$$

In order to use a hyperelastic model in an implicit finite element solver (e.g. ABAQUS/Standard [33]), the fourth order elasticity tensor has to be derived. In the reference configuration, the elasticity tensor is defined as

$$
\mathbb{C}=\frac{\partial^{2} W}{\partial \mathbf{E}^{2}}=\frac{\partial \mathbf{S}}{\partial \mathbf{E}}
$$

Substituting the Eq.10 into Eq.12 gives:

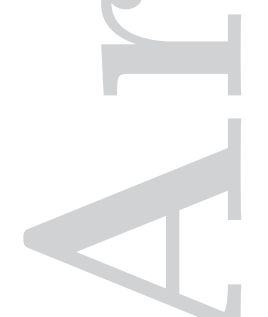

$$
\begin{aligned}
\mathbb{C} & =K J(J-1)\left(\mathbf{C}^{-1} \otimes \mathbf{C}^{-1}-2 \mathbb{I}_{\mathbf{C}^{-1}}\right)+K J^{2} \mathbf{C}^{-1} \otimes \mathbf{C}^{-1} \\
& +2 \mu \mathbb{I}+2 A \mathbf{E}: \mathbb{I}+8 D \mathbf{E} \otimes \mathbf{E}+4 D \operatorname{tr}\left(\mathbf{E}^{2}\right) \mathbb{I}
\end{aligned}
$$

where $\mathbb{I}=\frac{1}{2}\left(\delta_{i k} \delta_{j l}+\delta_{i l} \delta_{j k}\right)$ stands for the fourth-order identity tensor, and $\left(\mathbb{I}_{\mathbf{C}^{-1}}\right)_{i j k l}=$ $-\frac{1}{2}\left(\mathbf{C}_{i k}^{-1} \mathbf{C}_{j l}^{-1}+\mathbf{C}_{i l}^{-1} \mathbf{C}_{j k}^{-1}\right)$.

The spatial elasticity tensor can be obtained by the push-forward operation defined as:

$$
\mathbb{C}_{i j k l}=\frac{1}{J} \mathbf{F}_{i I} \mathbf{F}_{j J} \mathbf{F}_{k K} \mathbf{F}_{l L} \mathbb{C}_{I J K L}
$$

Particularly, ABAQUS/Standard uses the elasticity tensor associated to the Jaumann objective rate of the Cauchy stress, which can be obtained by:

$$
\mathbb{C}^{\sigma J}=\mathbb{C}+\frac{1}{2}\left(\delta_{i k} \sigma_{j l}+\sigma_{i k} \delta_{j l}+\delta_{i l} \sigma_{j k}+\sigma_{i l} \delta_{j k}\right) \mathbf{e}_{i} \otimes \mathbf{e}_{j} \otimes \mathbf{e}_{k} \otimes \mathbf{e}_{l}
$$

\subsection{Comparison}

In the above, one can see that continuum mechanics models and non-linear acoustic models use different invariants definitions of finite deformation tensors. It is of interest to illustrate the relationship between these models. Especially, it has been shown in [29, 10], that Rivlin and Landau's energy functions are equivalent upto the fourth order with the following relationships between the parameters:

$$
\mu=2\left(c_{10}+c_{01}\right), A=-8\left(c_{10}+2 c_{01}\right), D=2\left(c_{10}+3 c_{01}+2 c_{20}\right)
$$

Rivlin model is inherently included in ABAQUS package, Landau's model is implemented by a user subroutine (UMAT) written in Fortran. In order to verify these relationships, 
FEM simulations are carried out. We use a single hybrid hexahedral element $(C 3 D 8 H$ in ABAQUS) of $1 \times 1 \times 1 \mathrm{~mm}^{3}$. The material parameters are chosen to be the measured value in mixed gel [34]. So $\mu=6.6 k P a, A=-37.7 k P a$ and $D=30 k P a$. Accordingly, the equivalent Rivlin model parameters are: $c_{10}=1.8875 \mathrm{kPa}, c_{01}=1.4125 \mathrm{kPa}$ and $c_{20}=4.4375 \mathrm{kPa}$. Moreover, to illustrate the difference, the neo-Hookean model is also presented with $c_{10}=\frac{\mu}{2}=3.3 \mathrm{kPa}$. To ensure the quasi incompressibility, the bulk modulus $K$ is set to $1 G P a$ in all the models.

\subsubsection{Uniaxial tension/compression}

The first test consists in a uniaxial compression/tension up to stretches $\lambda$ of 0.5 to 1.5. The deformation gradient $\mathbf{F}$ is then written as

$$
\mathbf{F}=\left[\begin{array}{ccc}
\lambda & 0 & 0 \\
0 & \frac{1}{\sqrt{\lambda}} & 0 \\
0 & 0 & \frac{1}{\sqrt{\lambda}}
\end{array}\right]
$$

Fig. 1 represents the Cauchy stress along the stretch direction during the deformation. All three models have the same behaviour at small deformations range. However, Landau's and Rivlin models harden strongly in large deformations, both in tension and in compression, whereas neo-Hookean is almost linear in the whole range of deformation. In large deformations, Landau's model exhibits a larger nonlinearity, especially in tension, than Rivlin model. Moreover, both models have different behaviours between tension and compression, which is particularly clear for Rivlin model. The dissymmetric behavior in tension and in compression has been observed in experiments on biological tissues [35].

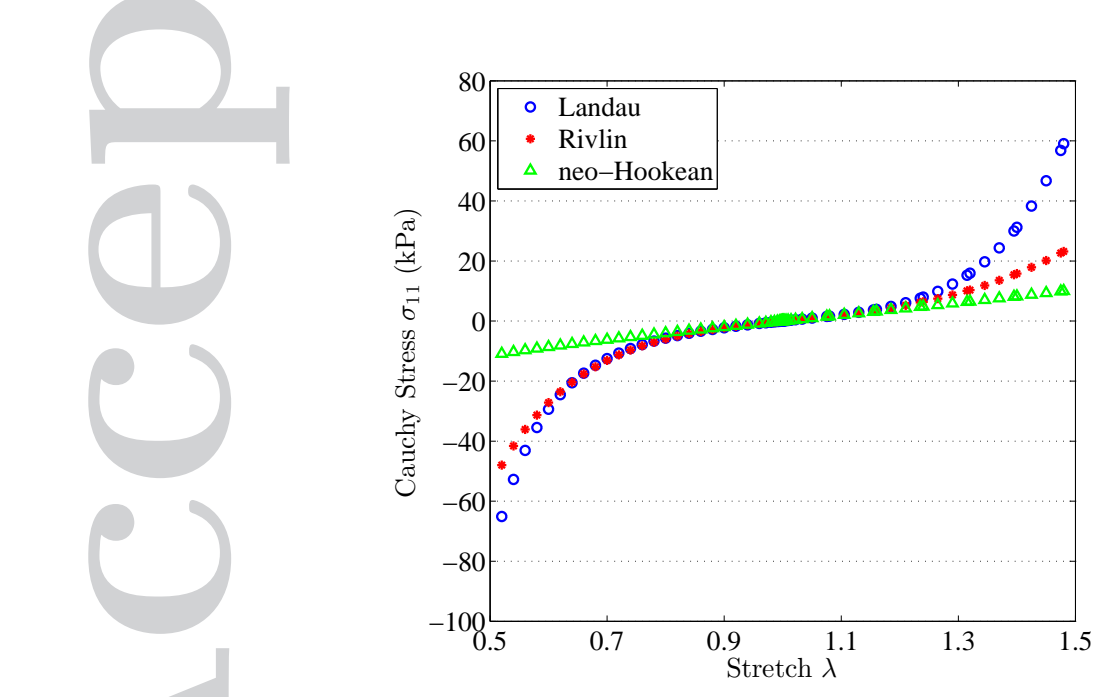

Figure 1. Uniaxial tension/compression test 


\subsubsection{Simple shear}

Now we consider an elastic cube sheared by two plates, the deformation gradient is given by:

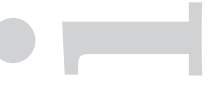

$$
\mathbf{F}=\left[\begin{array}{lll}
1 & 0 & 0 \\
0 & 1 & \kappa \\
0 & 0 & 1
\end{array}\right]
$$

with $\kappa$ is the amplitude of shear strain which varies from 0 to 0.5 .

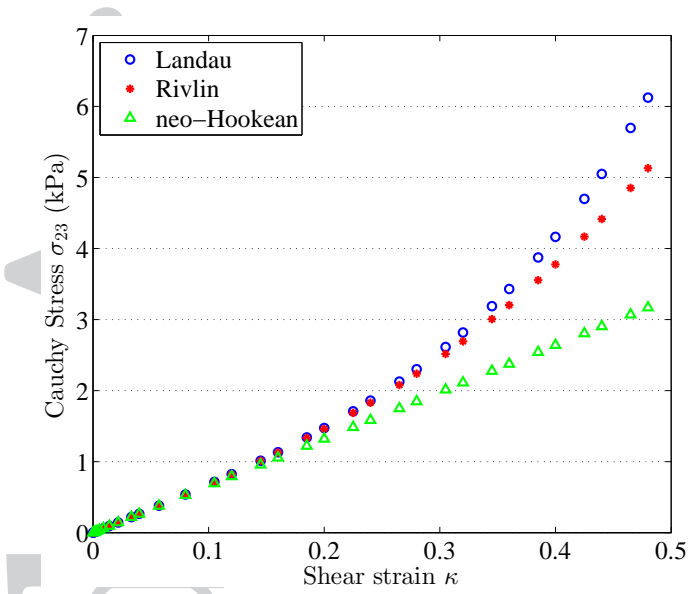

(a) Shear stress

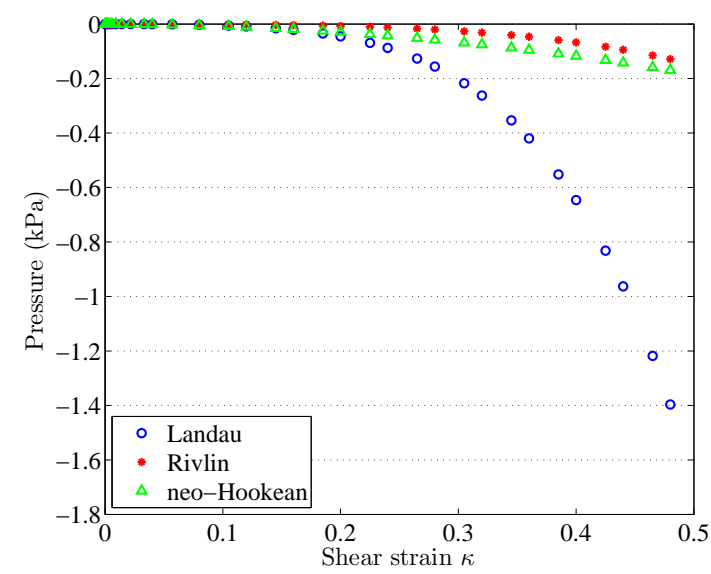

(b) Pressure

Figure 2. Simple shear test. Fig.2(a) shows the relation between the shear strain and the shear stress; Fig.2(b) shows the pressure in simple shear which reflects Kelvin effect

Fig.2(a) displays the shear strain-stress curves. For the three models, the behaviours are similar at the beginning of the load because of the same initial shear modulus $\mu$. Then, Landau's and Rivlin models exhibit moderate hardening at large strains. However, there is still an obvious difference in large shear, up to $20 \%$ when $\kappa$ equals 0.5 . On the other hand, neo-Hookean law has an exactly linear behaviour. This property being essential for the nonlinear shear wave propagation study in the following (Section 3), the analytical expression of shear stress as a function of strain $\kappa$ is given in Appendix A.

Fig.2(b) represents the pressure evolution during shear deformation. Although there is no volume change in this case, pressure is still generated, this is known as Kelvin effect [36]. It can be seen that this effect is much stronger in Landau's model than in the other two models, nevertheless, the pressure is much smaller than shear stress at a given deformation.

To sum up, the behaviours of three different hyperelastic models are compared in simple cases. Importantly, the similarity and difference between Rivlin and Landau's model have been shown. This comparison highlights the need for developing accurate material models for numerical modeling. For our purpose (simulating non-linear wave propagations in soft 
solids), the material model should be carefully considered. In limited deformations, Rivlin and Landau's model have almost the same behaviour by using the relationships Eq.16. In large deformations, Rivlin model is less non-linear than Landau's model which is due to the 4th order truncation of Rivlin model. However, this may be out of the range of validity of weakly non-linear theory (Landau's law). Finally, models such as neo-Hookean do not have non-linear behaviour in shear.

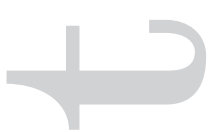

\subsection{Voigt viscoelasticity}

In non-linear dynamic simulations of soft solids, materials cannot be accurately modeled without considering viscosity. For finite amplitude waves, non-linear elasticity generates higher harmonics of the fundamental frequency when the wave propagates, meanwhile, the absorption of the medium decreases the amplitude of deformation which limits the generation of these harmonics. In this subsection, the development of a non-linear viscoelastic model which is based on Landau's law and Voigt model is described.

Voigt model is chosen because it describes accurately the viscous property of soft solids at low frequency range, which is consistent with the typical excitation of transient elastography, i.e., $50-200 \mathrm{~Hz}$. Moreover, this model is verified in the experimental work for both artifical and biological tissues [37].
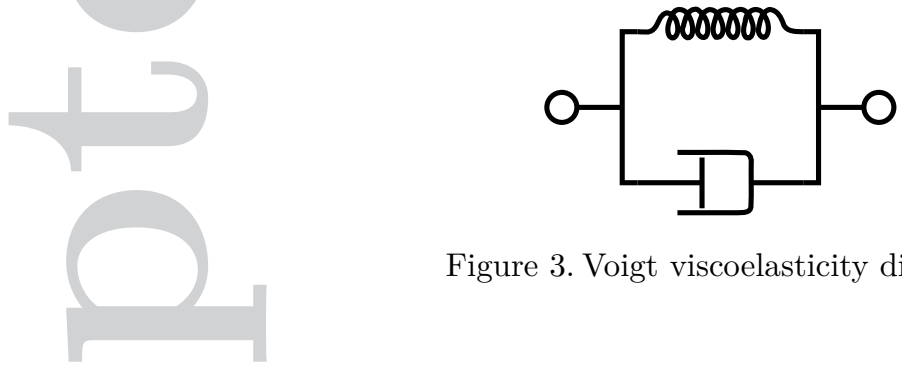

Figure 3. Voigt viscoelasticity diagram

In infinitesimal strain theory, Voigt model has been described in numerous books [38, 39]. Here, only its generalization to finite strain is presented. Non-linear Voigt material is also represented by a purely viscous damper and purely elastic spring connected in parallel, as shown in Fig.3. The total stress is the sum of the elastic stress and viscous stress:

$$
\mathbf{S}^{\text {tot }}=\mathbf{S}^{\text {elas }}+\mathbf{S}^{\text {visco }}
$$

In the above expression, PK-2 stress $\mathbf{S}$ is employed, but the expression is also available for Cauchy stress $\boldsymbol{\sigma}$ if the FEM code is based on the current configuration. In our work, Landau's law is considered in the elastic part, so $\mathbf{S}^{\text {elas }}$ is calculated by Eq.10. 
The non-linear viscosity has been considered in [11]. Under the assumption of isotropy, the viscous stress $\mathbf{S}^{\text {visco }}$ is written in terms of deviatoric and volumetric part as for the elastic stress, that is,

$$
\mathbf{S}^{v i s c o}=2 \eta \dot{\mathbf{E}}^{d e v}+\xi \dot{\mathbf{E}}^{\text {vol }}
$$

where the superposed dot denotes the time derivative, $\eta$ and $\xi$ are the shear and bulk viscosity coefficients, respectively.

It can be seen that this is the same treatment as in infinitisimial strain. However, it is essential that the correct work conjugate stress and strain measures be used when considering finite strain [40]. The decomposition of the rate of the Green strain tensor $\dot{\mathbf{E}}$ into deviatoric and volumetric parts is presented in [39]:

$$
\dot{\mathbf{E}}^{v o l}=\frac{1}{3}\left(\dot{\mathbf{E}}: \mathbf{C}^{-1}\right) \mathbf{C}, \quad \dot{\mathbf{E}}^{d e v}=\dot{\mathbf{E}}-\dot{\mathbf{E}}^{v o l}
$$

Finally, Landau's viscoelastic model is implemented in our in-house FE code and it will be employed in the numerical simulations presented in the following.

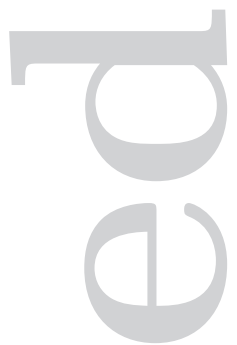

\section{SIMULATIONS OF NON-LINEAR SHEAR WAVE}

In this section, non-linear shear wave propagation in soft solids is analyzed using FEM simulations. Two models are considered, one for plane shear waves and the other for nonplane shear waves, they are both based on experiments of artificial gels in [1] . In this experiment, it has been observed that a plane wave generates only the odd harmonics at $3 f, 5 f, \ldots$ ( $f$ is the fundamental frequency), and a nonplane shear wave generates all harmonic components, i.e. $2 f, 3 f, \ldots$ For plane waves, the phenomenon can be explained as the cubic term in the shear stress expression (Eq.30) contains only the odd frequencies after the Fourier transform [6]. However, there is still no analytical model for nonplane wave. The objective of this section is to illustrate that FEM is able to simulate both plane and nonplane waves and to provide very similar results to both the analytical model and the experiments.

In the following, simulations are carried out by our in-house FE code, Landau's viscohyperelastic model presented above is employed. In numerical aspect, bi-linear quadrangular elements combined with selective integration strategy are used to handle volumetric locking and hourglass effect at the same time. Besides, an explicit scheme is employed because it is 
the most suited time integration method for wave propagation problems [41], even though its efficiency is decreased by the high incompressibility of soft solids.

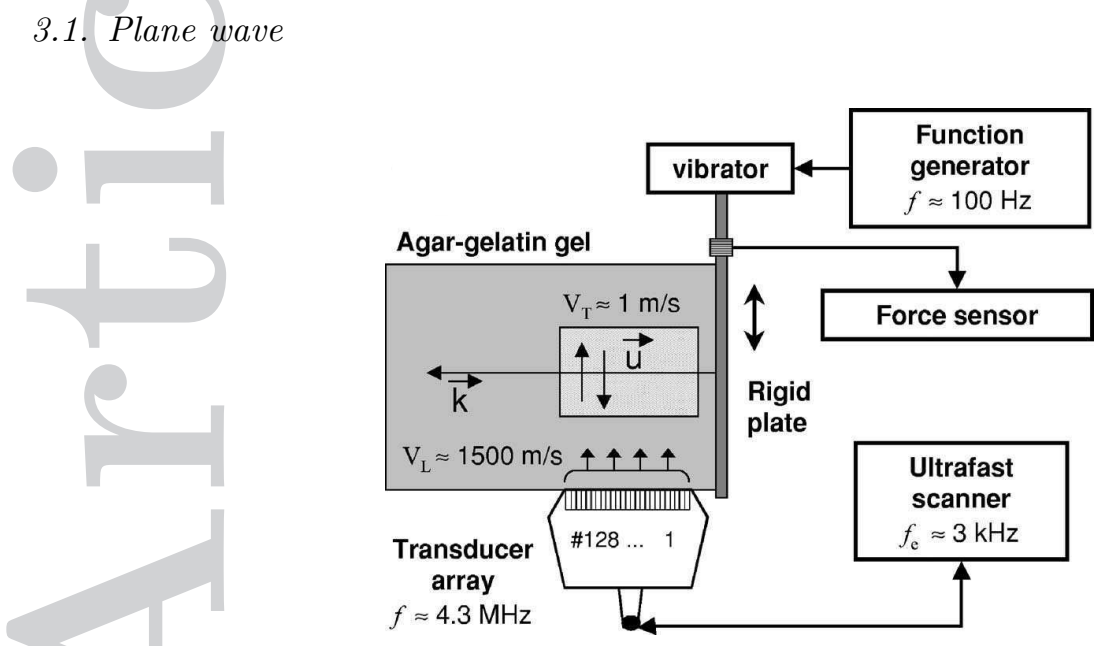

Figure 4. Non-linear plane shear wave experiment presented in [1].

The propagation of plane shear waves is considered firstly. In the experiment, the artifical solid is composed of agar and gelatin mixed with hot water and solidified. A rigid plate is placed on the right side of the solid and generates a low frequency shear wave, see Fig.4. The shear wave propagates transversely at a very low speed (several meters per second), and this particle motion is measured by a transducer array placed at the bottom. For a more detailed description, the reader is invited to consult the original paper [1].
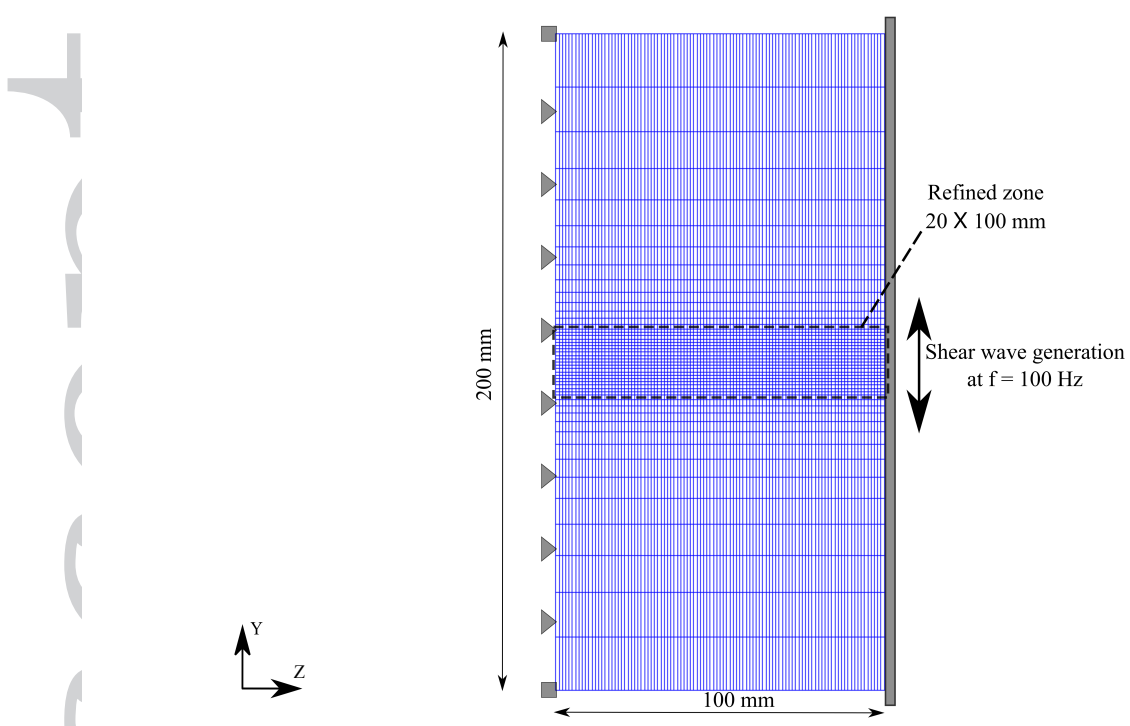

Figure 5. Geometry and FE model of plane shear wave propagation simulation.

The geometry and the FE model for the plane shear wave experiment are shown in Fig.5. The plane strain hypothesis can be used, which allows an enormous reduction of the 


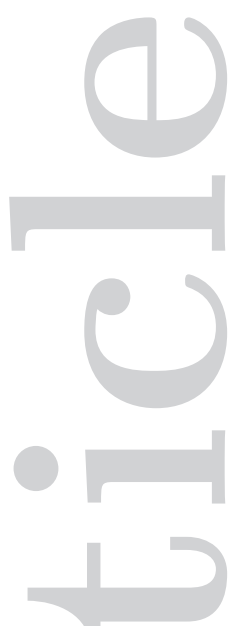

\begin{tabular}{lc}
\hline \multicolumn{2}{c}{ Material parameters } \\
\hline$\rho\left(k g / m^{3}\right)$ & 1000 \\
$\mu(k P a)$ & 4.4 \\
$A(k P a)$ & 41.1 \\
$D(k P a)$ & 0 \\
$K(k P a)$ & $10^{4}$ \\
$\eta(k P a s)$ & $0.6 \times 10^{-3}$ \\
$\xi(k P a s)$ & $0.5 \times 10^{-5}$ \\
\hline
\end{tabular}

Table I. Material parameters of Landau's visco-hyperelastic law.

computation cost. The size of the model is chosen to be large enough to avoid reflections, hence the model contains 4949 nodes and 4800 elements. The measurement is only located in the zone where the mesh has been refined by 2000 square elements. The length of each element is $1 \mathrm{~mm}$ which ensures more than 20 elements per wavelength $\left(c_{s} / f\right)$.

The material parameters of Landau's visco-hyperelastic law are summarized in Tab.I, they are chosen from the values reported in the experiments. In order to keep the time step reasonable in numerical simulations, the bulk modulus $K$ is set to $10^{4} k P a$ which is about two orders smaller than its real value. However, it is still three orders larger than the shear modulus $\mu$, so relative incompressibility is ensured. Convergence study of this approximation has been given in [17]. Besides, it should be emphasized that only a relation of the third and fourth order shear moduli $A$ and $D$ is given in [1], there is no effective way to discriminate them separately. Herein, we set $D=0$ to determine the value of $A$. In the Section 4 , it will be shown that this approximation has minor effect in the results.

The boundary conditions are set to prevent the rigid motion, see Fig.5. To generate a plane wave, a vertical displacement is applied on the right side of the model, the dynamic excitation is described by the following function:

$$
U_{y}(t)=A_{0} \sin (2 \pi f t)\left(1-\cos \left(\frac{2 \pi f}{N} t\right)\right), \quad 0 \leqslant t \leqslant \frac{N}{f}
$$

where $A_{0}=0.8 \mathrm{~mm}, N=5$ and the frequency $f=100 \mathrm{~Hz}$.

This simulation lasts for $0.075 \mathrm{~s}$, the time step is set to $0.5 \times 10^{-6} \mathrm{~s}$ to keep the stability of the explicit scheme.

Fig.6 shows the vertical displacement distribution in the refined zone at three times. It can be observed that the wave front remains plane until the end of the simulation, which indicates that the assumption of plane wave propagation is hold.

The resulting velocity of the source and the corresponding spectrum are displayed in Fig.7 with blue lines. The source contains only the component of $100 \mathrm{~Hz}$, which is the fundamental component. The maximal velocity reaches $500 \mathrm{~mm} / \mathrm{s}$, it is about $24 \%$ of the linear shear 
wave speed in this model $\left(c_{s}=2.1 \mathrm{~m} / \mathrm{s}\right)$. This ratio ensures that the non-linear behaviour can be clearly observed.

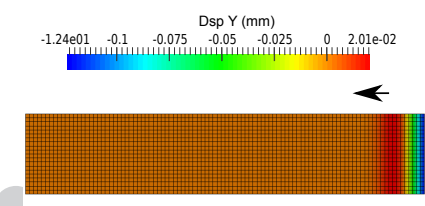

(a) $t=0.0075 \mathrm{~s}$

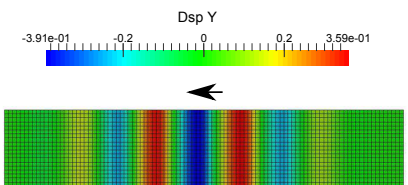

(b) $t=0.0525 \mathrm{~s}$

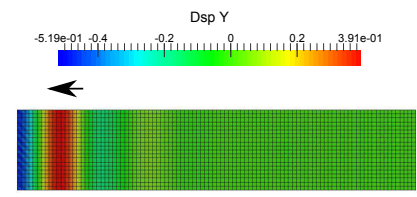

(c) $t=0.075 \mathrm{~s}$, end of simulation

Figure 6. Vertical displacement distributions in the refined zone. The wave front remains plane during the simulation; it confirms the hypothesis of plane shear wave propagation

In the numerical results, the vertical velocity history and its spectrum are measured $20 \mathrm{~mm}$ away from the source, see the red lines in Fig. 7. In the time domain, the wave form is clearly different from the source signal, the distortion is in a dis-symmetrical way which is reflected by the odd harmonic components $(300,500 \mathrm{~Hz}, \ldots)$ in the spectral domain.
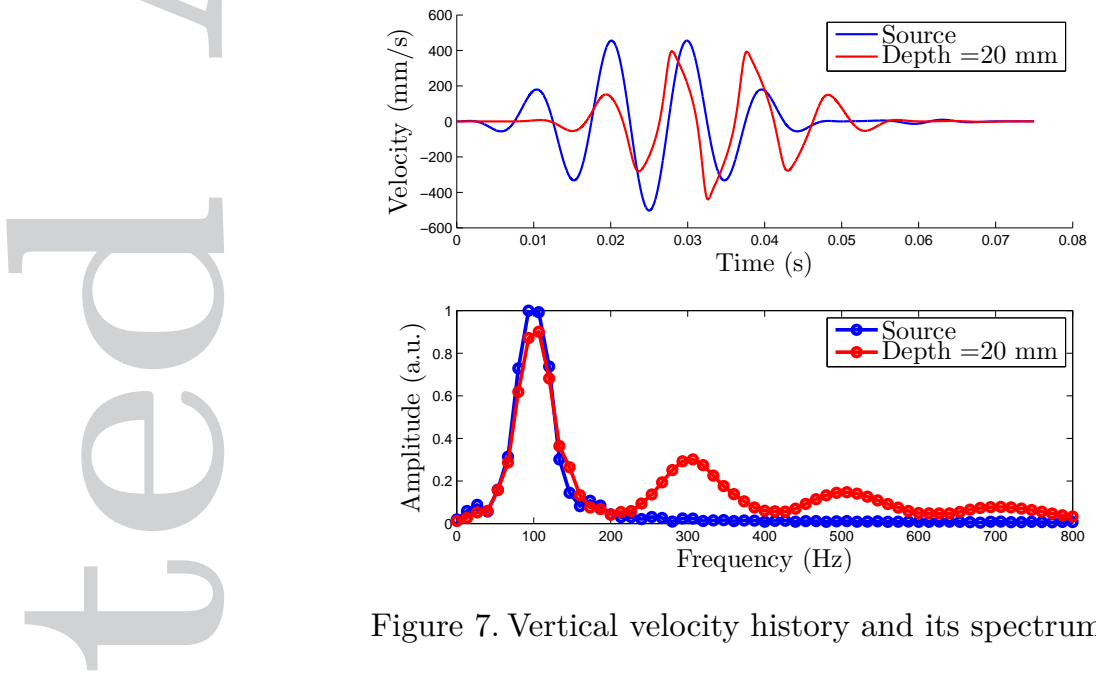

Figure 7. Vertical velocity history and its spectrum

A comparison with the experimental results of [1] is displayed in Fig.8. The figure represents the global spectrum along the distance of propagation from 5 to $50 \mathrm{~mm}$. It can be seen that two results are very close, both the fundamental and the first odd harmonic $(3 f)$ cross the whole model with similar amplitudes.

The evolutions of these amplitudes are presented as a function of the depth, and displayed in Fig.9. In the numerical results (Fig.9(a)), the fundamental component (100 Hz) decreases linearly because of the viscosity. The two harmonic components reach $10 \%$ and $3 \%$ of the fundamental component respectively. The maximal amplitudes locate at the depth of $12 \mathrm{~mm}$, it reflects the fact that a part of the energy is transmitted to higher frequencies because of the elastic nonlinearity. Moreover, one can see that the harmonics amplitude grows until $12 \mathrm{~mm}$ and then decreases, the tendency is due to the fact that the viscous dissipation becomes the dominant effect with respect to the nonlinearity. The experimental results are 


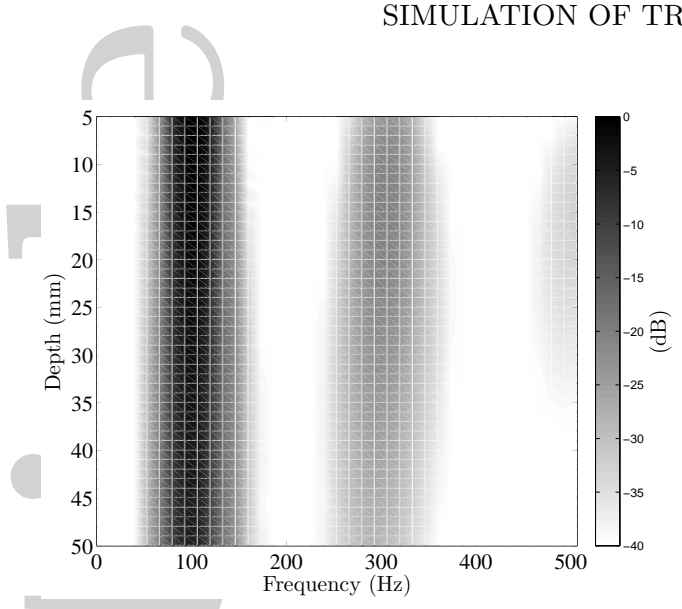

(a) Simulation results

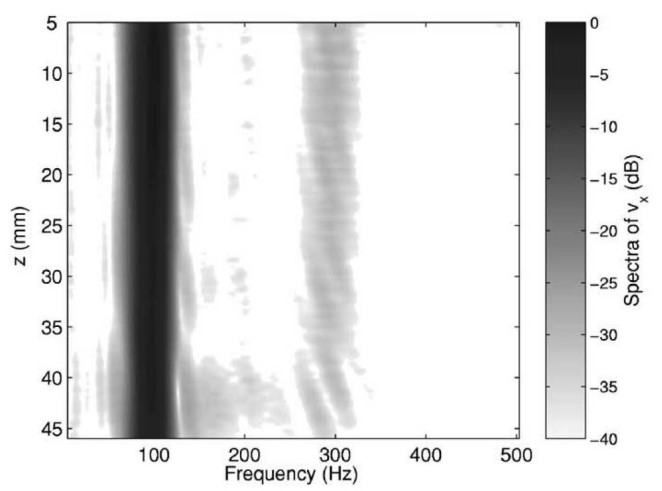

(b) Experimental results in [1]

Figure 8 . Spectra of the vertical velocity along the propagation depth $z$

shown in Fig.9(b), the harmonic components seem slightly larger, however, the two results are still very similar.

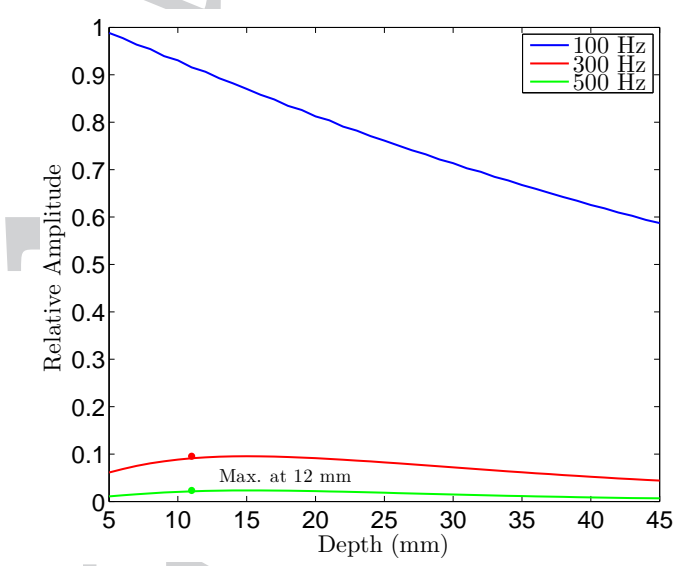

(a) Numerical simulation results

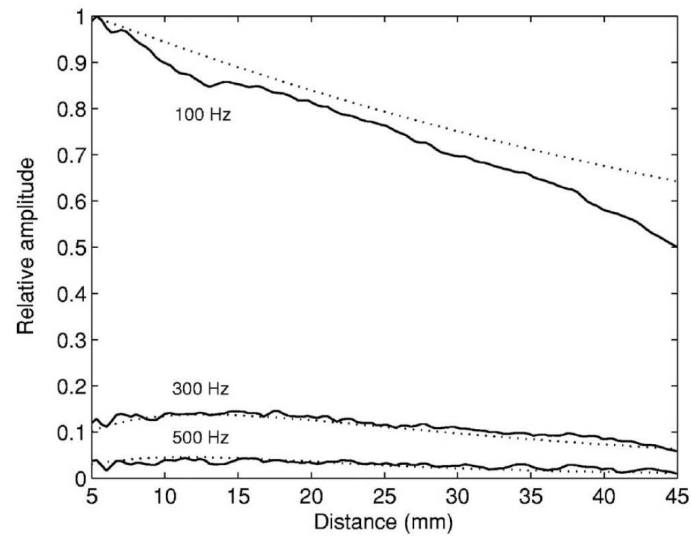

(b) Experimental results (solid lines) in [1] (The dotted lines present the analytical solution)

Figure 9. Plane wave: Evolution of the fundamental component and the third and fifth harmonics along the propagation depth

\subsection{Nonplane wave}

In this part, nonplane shear wave propagation is investigated for the same material. The corresponding experimental setup is presented in Fig.10(a). In this model, the propagation direction $(\vec{k})$ coincides with the polarization direction $(\vec{u})$, the wave is refered to longitudinallike polarized shear wave, and it has been theoretically predicted in the linear domain [42].

The experiment shows that this shear wave generates all harmonics components (at $2 f$, $3 f, \ldots)$. To our knowledge, an analytical method is still missing to predict this phenomenon, so the numerical model could be a valuable tool to validate this experiment. 


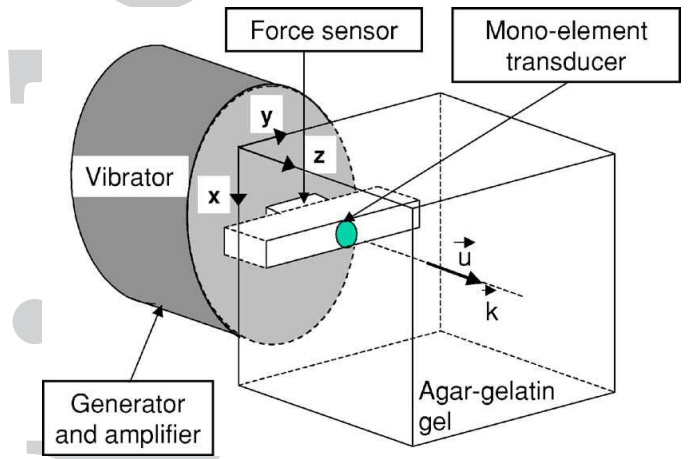

(a) Experiment setup in [1]

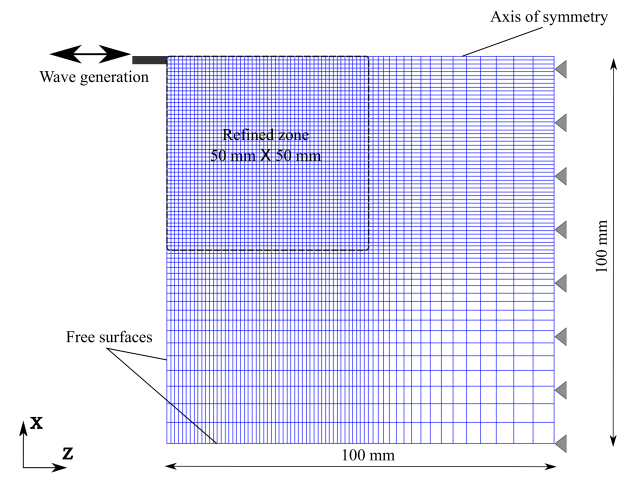

(b) FE model

Figure 10. Nonplane shear wave model. As both the gel and the vibrator are set to be enough large in the experiment, EF model can take advantage of plane strain hypothesis.

In this experiment, both the solid and the vibrator bar are large enough to avoid reflections from the two lateral sides, so the plane strain hypothesis can be used again. The finite element model is described in Fig.10(b), it has 4900 nodes and 4761 elements, the refined zone contains 2500 squares with a length of $1 \mathrm{~mm}$. The vibration bar is modelled by prescribing the displacement at the top-left corner on the width of $2 \mathrm{~mm}$. The measurement locates along the symmetric axis of the refined zone, the nodal velocity in the $z$ direction is recorded at every time step.
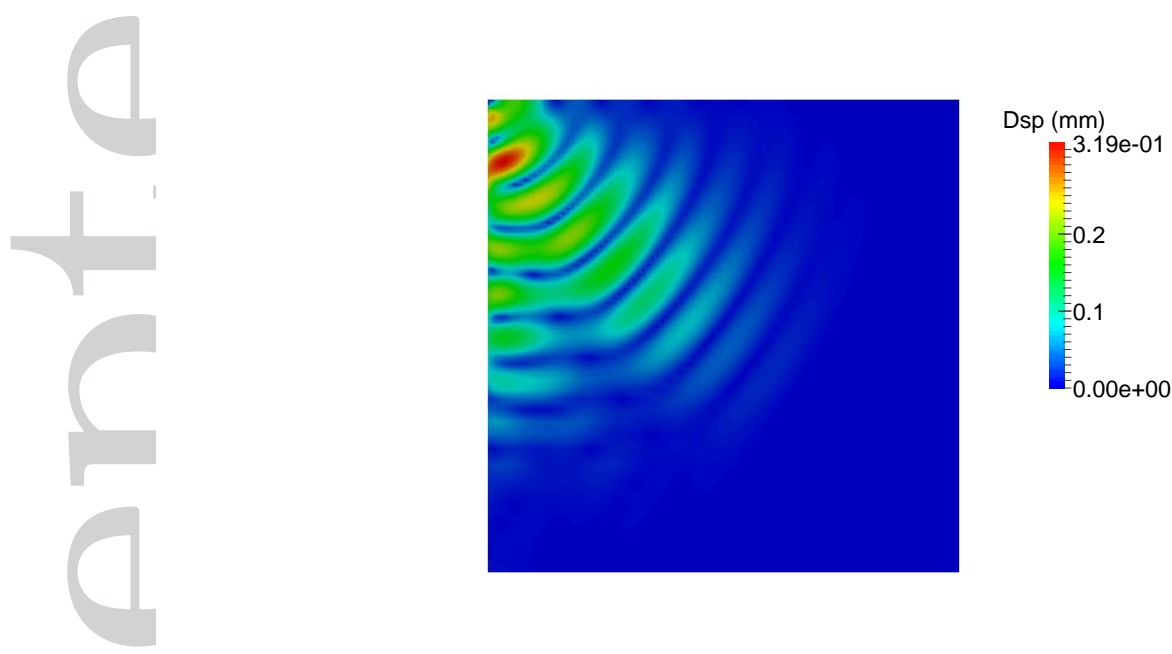

Figure 11. Nonplane wave model: displacement distribution at $t=0.05 \mathrm{~s}$

The material parameters have been summarized in Tab.I. The excitation is also described by Eq.22, with $A_{0}=0.8 \mathrm{~mm}, N=7$ and $f=100 \mathrm{~Hz}$. Fig.11 shows the displacement contour at time $t=0.05 \mathrm{~s}$, both the shear wave front and the surface wave front (at the left side) emitted by the source can be observed. The foregoing wave front does not reach the edge of the model, so the measurement can not disturbed by reflections. 
In Fig.12, the particles velocity and the corresponding spectrum of the source and at two different points are compared. Unlike the plane waves, the wave form remains symmetric along the propagation depth, and all (odd and even) harmonics components $(2 f, 3 f, \ldots)$ appear in the spectrum. Then, the global spectrum is displayed in Fig.13, the harmonic components are still observable, however, we can see that the amplitude decreases quickly as long as the wave propagates, which reflects the diffraction caused by the finite size of the source. These results are very similiar to the experimental observation in [1].
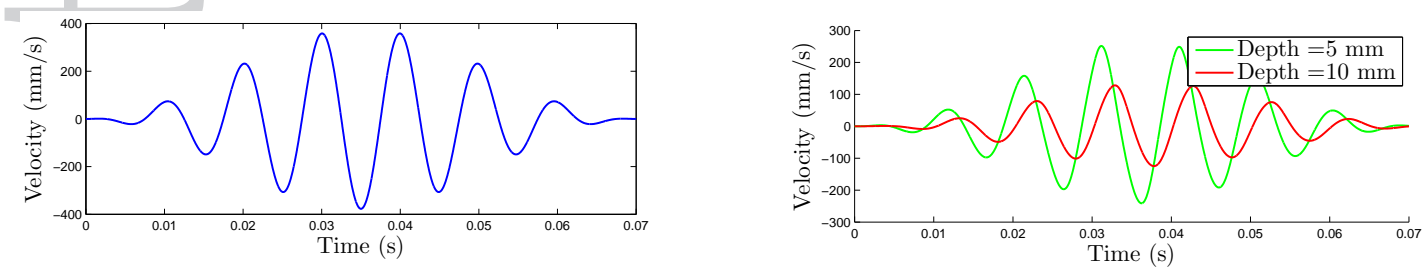

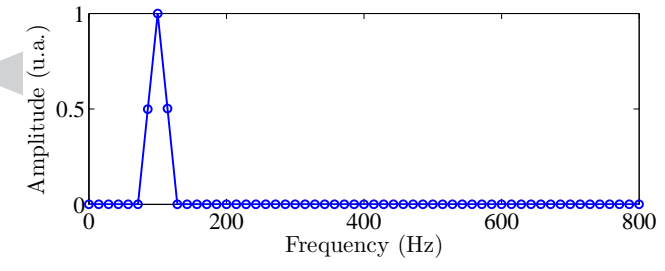

(a) Source velocity and spectrum

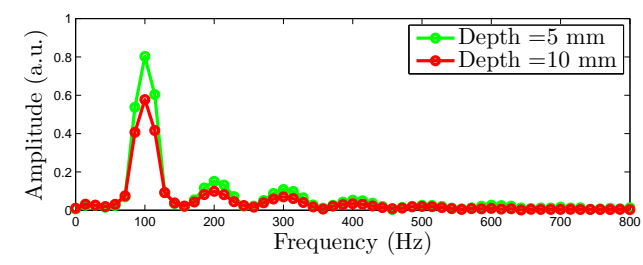

(b) Particle velocity and its spectrum at different depth

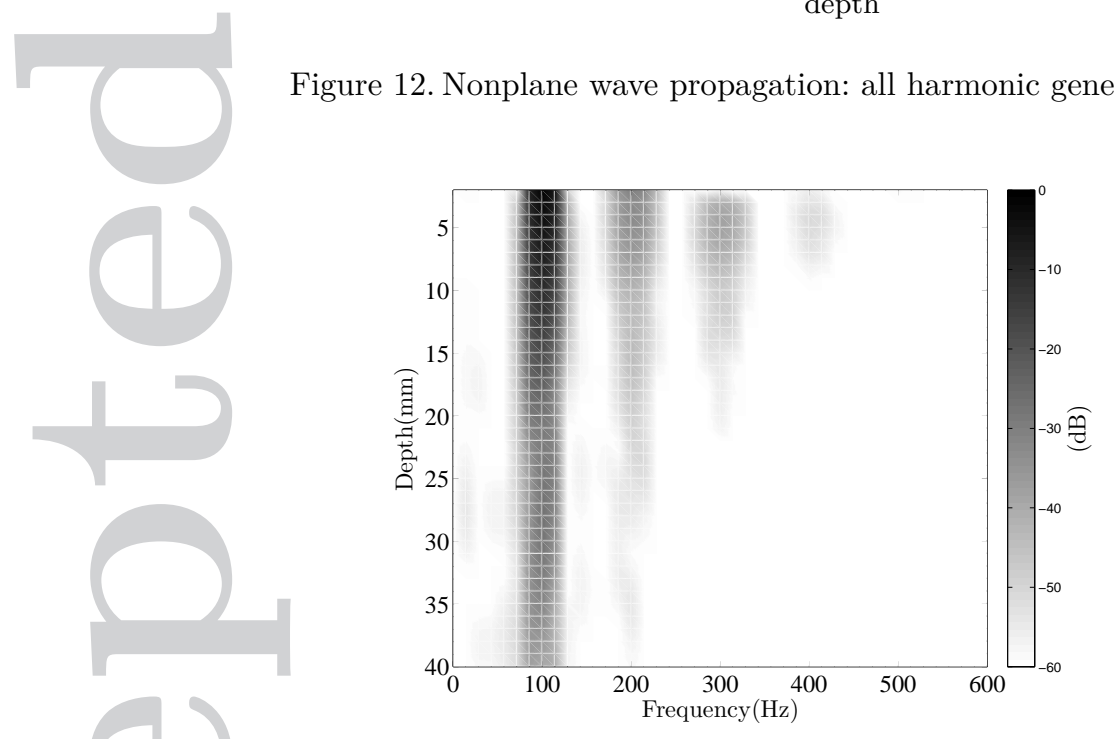

Figure 13. Global spectrum of nonplane wave propagation

\subsection{Discussion}

In this section, FEM simulations of non-linear shear wave propagation in soft solids are presented. Both plane and nonplane shear waves are generated by a harmonic excitation, the numerical results show a good agreement with the experimental work.

In [1], the experiments with two superimposed excitations have also been reported, the results show that the non-linear interaction between two waves generates more harmonics. 
However, the corresponding simulations are not shown here because the mechanism is the same as those observed for the single harmonic excitation.

In addition, the same finite element analysis have been carried out for neo-Hookean and Rivlin models, the viscosity is considered the same way (Voigt model). As predicted in Section 2, a neo-Hookean model produces no harmonics because of its linear behaviour in shear, and Rivlin model produces the same harmonics as Landau's law because the two models are identical at the range of deformation produced by non-linear waves.

\section{PARAMETRIC STUDY}

In the above, we have shown that non-linear elasticity generates higher harmonics. At the same time, viscosity has two effects: firstly, it decreases the amplitude of wave which limits the continuing generation of the harmonics. Secondly, the absorption coefficient increases with frequency, so the higher harmonics attenuate quickly which leads to another energy loss.

The phenomenon of harmonics generation can provide a guideline to identify the nonlinearity of soft solids by transient elastography. In this section, a parametric study is carried out for the plane wave model, the objective is to find a practical method to identify the non-linear elastic parameters ( $A$ or $D$, or both) of homogeneous tissues.

\subsection{Analytical formulation}

Before performing the parametric study by FEM, we recall the analytical formulation given by Zabolotskaya et al. [11] for fundamental and first odd harmonic amplitude. Given a harmonic source excitation, by supposing the harmonic component remains small, the fundamental and first odd harmonic amplitude (of particular velocity) as a function of the propagation distance $z$ can be expressed as follow:

$$
\begin{aligned}
& \bar{A}_{1}(z)=v_{0} e^{-\alpha z} \\
& \bar{A}_{3}(z)=-\frac{\beta \omega v_{0}^{3}}{24 c_{s}^{3} \alpha}\left(e^{-3 \alpha z}-e^{-9 \alpha z}\right)
\end{aligned}
$$

These equations involve: the shear absorption coefficient $\alpha=\frac{\eta \omega^{2}}{2 \rho c_{s}^{3}}$, the dimensionless nonlinear elastic coefficient $\beta=\frac{3 \gamma}{2 \mu}$, the angular frequency $\omega=2 \pi f$ and the source velocity $v_{0}=A_{0} \omega$. 


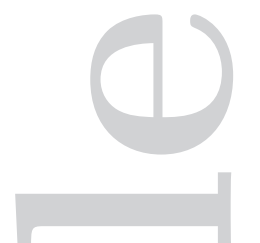

\begin{tabular}{lc}
\hline \multicolumn{2}{c}{ Material parameters } \\
\hline$\rho\left(k g / m^{3}\right)$ & 1000 \\
$\mu(k P a)$ & 4 \\
$A(k P a)$ & 40 \\
$D(k P a)$ & 0 \\
$K(k P a)$ & $10^{4}$ \\
$\eta(k P a s)$ & $0.3 \times 10^{-3}$ \\
$\xi(k P a s)$ & $0.5 \times 10^{-5}$ \\
\hline Load parameters $($ Eq.22) \\
\hline$f(H z)$ & 100 \\
$A_{0}(m m)$ & 0.6 \\
$N$ & 5
\end{tabular}

Table II. Referenced model used in parametric study

It should be emphasized that this analytical result is obtained under the assumption of weak nonlinearity, which assumes that Goldberg number $\Gamma$ is small. Goldberg number is a dimensionless coefficient to characterize the relative importance of nonlinearity and viscosity. For a harmonic excitation, it can be written as:

$$
\Gamma=\frac{2 \rho \beta v_{0}^{2}}{\eta \omega}
$$

The relative amplitudes are used in the following for comparison purpose. They are defined by:

$$
\begin{aligned}
& A_{1}(z)=\overline{A_{1}}(z) / \max \left(\overline{A_{1}}\right) \\
& A_{3}(z)=\overline{A_{3}}(z) / \max \left(\overline{A_{1}}\right)
\end{aligned}
$$

This analytical model has been used in [34] to identify the non-linear elastic coefficient of mixed gel. However, the model has two limits. Firstly, the second harmonic component at $5 f$ has not been considered. Secondly, Eq.23 is only valid for small Goldberg numbers which limits its application in real elastography experiments.

These limits can be mended by the numerical simulations. In the following, the plane wave model presented in Section 3.1 is employed again, all parameters involved in Goldberg number (Eq.24), i.e. amplitude, frequency, viscosity, nonlinearity, are studied separately. In each analysis, without an opposite statement, the simulation parameters (summarized in Tab.II) remain the same as in Section 3.1.

\subsection{Effect of amplitude}

Firstly, the effect of the load amplitude $A_{0}$ is investigated. At the same frequency, the higher the amplitude, the more nonlinearity is observed. We choose for the souce amplitude $A_{0}$ in 
Eq.22 the values of $0.4 \mathrm{~mm}, 0.6 \mathrm{~mm}$ and $0.8 \mathrm{~mm}$, other simulation parameters are kept constant. So the Goldberg numbers are 6.03, 13.57, 24.13 respectively by Eq.24.

The relative amplitude at the fundamental frequency and two harmonics are presented in Fig.14. At the fundamental frequency, as seen in Fig.14(a), dissipation is linear and increases with increasing amplitude. Nonlinearity effect is higher so more energy is transmitted into the higher frequencies domain.

The first odd harmonic component is displayed in Fig.14(b), and the analytical results by Eq.23 are also presented. In the case of a small Goldberg number (blue), the analytical solution shows a good agreement with the simulation result. As the Goldberg number grows, the analytical solution quality decreases. In Fig.14(c), the second harmonic amplitude is shown, notice that the maximum amplitude locations change slightly with influence of the load amplitude.

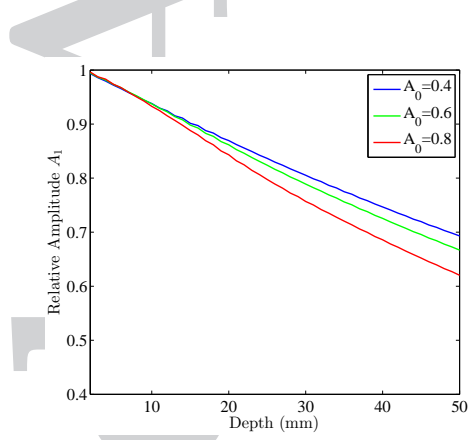

(a) At fundamental frequency $f$

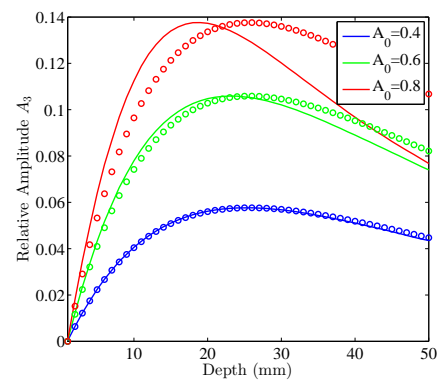

(b) At first odd harmonic $3 f$

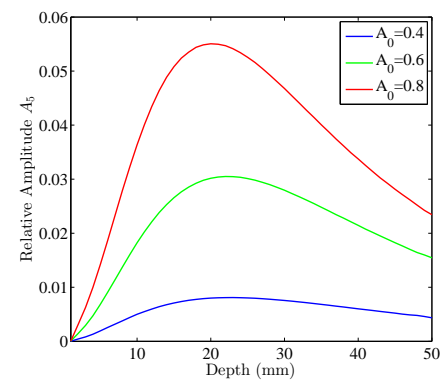

(c) Second odd harmonic: $5 f$

Figure 14. FEM simulations at different amplitudes: Evolution of the fundamental and odd harmonics components along the propagation distance. Note: circle marks in the Fig.(b) are the analytical results by Eq.23.

\subsection{Effect of frequency}

The second test consists in using different excitation frequencies but the same load amplitude. In this case, the non-linear wave is generated at the frequency of $80 \mathrm{~Hz}, 100 \mathrm{~Hz}$ and $120 \mathrm{~Hz}$, they lead to Goldberg numbers of 10.86, 13.57, 16.29 respectively. Note that different frequencies change the source velocity, and also the dissipation character.

The results are shown in Fig.15. In Fig.(a), the dissipation effect increases very quickly with the frequency. The first harmonic amplitudes are shown in Fig.(b). Again, the analytical solution leads to a good agreement with the numerical solution when Goldberg number is small. Then, it can be seen that high frequency excitation generates also high harmonic amplitudes. But contrary to the effect of amplitude, the frequency of excitation changes largely the location of the maximal amplitude. At high frequency, the harmonics build up earlier. 


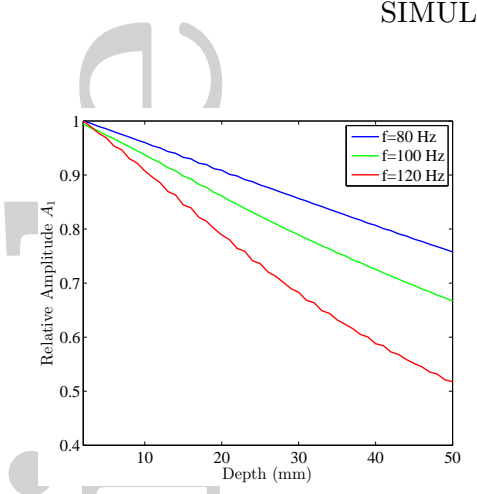

(a) At fundamental frequency

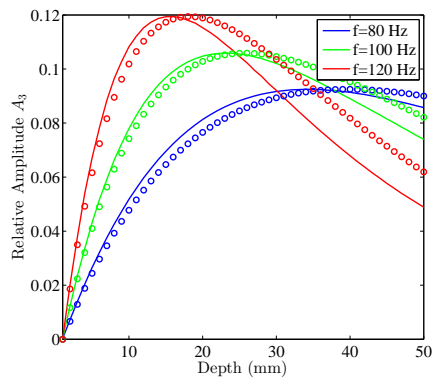

(b) At first odd harmonic: $3 f$

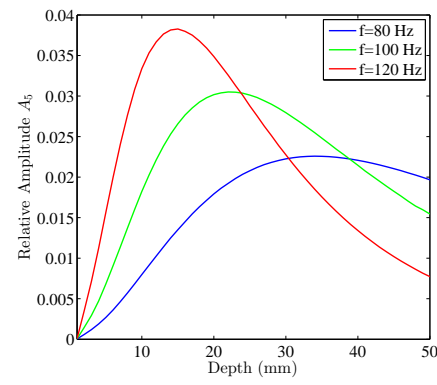

(c) At second odd harmonic: $5 f$

Figure 15. FEM simulations at different frequencies: Evolution of the fundamental and odd harmonics components along the propagation distance. Note: circle marks in the Fig.(b) are the analytical results by Eq.23.

\subsection{Effect of viscosity}

The effect of the viscosity is now considered. Three shear viscous coefficients $\eta$ are chosen, they are 0.2, 0.3 and $0.4 P a s$. Consequently, the Goldberg numbers are 20.36, 13.57, 10.18.

As shown in Fig.16, a small viscous coefficient leads to less loss of the fundamental component, but creates higher harmonic amplitudes. Also, it makes the maximal harmonics amplitude set up later. As expected, the analytical solution is valid when Goldberg number is small. Furthermore, in Fig.16(c), it can be seen the second harmonic amplitude with less viscosity (blue curve) is much more outstanding. It can be explained by the fact that the viscosity coefficient has more effect at high frequency than at low frequency.

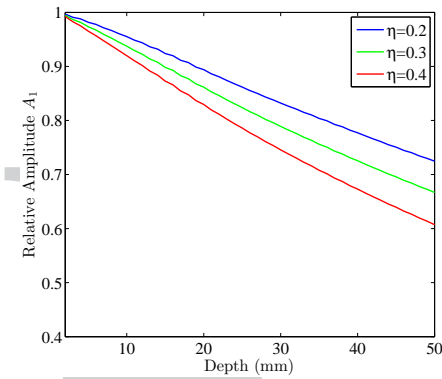

(a) At fundamental frequency

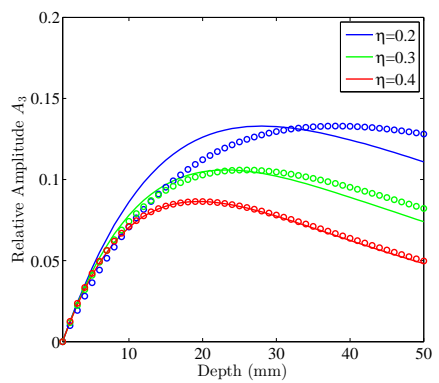

(b) At first odd harmonic: $3 f$

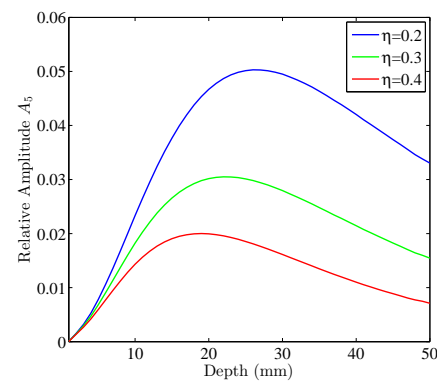

(c) At second odd harmonic: $5 f$

Figure 16. FEM simulations with different viscosities: Evolution of the fundamental and odd harmonics components along the propagation distance. Note: circle marks in the Fig.(b) are the analytical results by Eq.23.

\subsection{Effect of the first non-linear elastic parameter $\gamma$}

Now, we verify the influence of the first non-linear elastic parameter $\gamma=\mu+\frac{A}{2}+D$ (Eq.31).

Three materials summarized in Tab.III are tested, it can be seen the fourth-order shear modulus $D$ is set to 0 . As other parameters remain the same, Goldberg numbers are 2.26, $7.92,13.57$ respectively. 


\begin{tabular}{lcccc}
\hline Material & $\mu$ & $A$ & $D$ & $\gamma$ \\
\hline No. 1 & 4 & 0 & 0 & 4 \\
No. 2 & 4 & 20 & 0 & 14 \\
No. 3 & 4 & 40 & 0 & 24 \\
\hline
\end{tabular}

Table III. Different materials in the parametric study of $\gamma$. Unity: $k P a$

\begin{tabular}{lccccc}
\hline Material & $\mu$ & $A$ & $D$ & $\gamma$ & $\zeta$ (Eq.32) \\
\hline No. 4 & 4 & 40 & 0 & 24 & 10 \\
No. 5 & 4 & 0 & 20 & 24 & 30 \\
No. 6 & 4 & 60 & -10 & 24 & 0
\end{tabular}

Table IV. Different combinations of elastic moduli in the parametric study of $\zeta$. Unity: $k P a$

Fig.17 shows the simulation results. The following conclusions are obtained within expectations. Firstly, in Fig.(a), the red line decreases more than the others because more energy is transmitted to generate harmonics components. Secondly, in Fig.(b)(c), it is clearly observed that the highly non-linear material generates larger harmonics amplitudes. But even when both $A$ and $D$ are 0 (Material No.1), Landau's material is still non-linear and small amplitude harmonics exist. Lastly, the analytical model gives the accurate results only in weak nonlinearity.

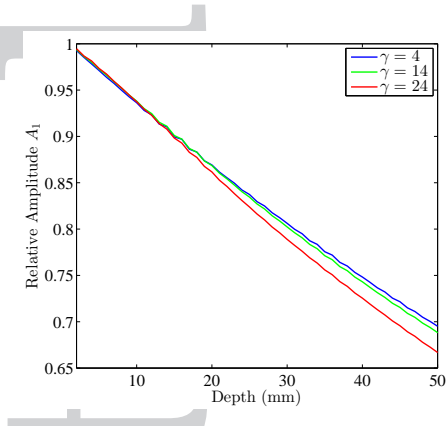

(a) At fundamental frequency $f$

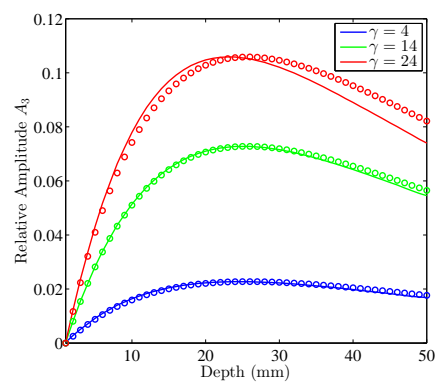

(b) At first odd harmonic $3 f$

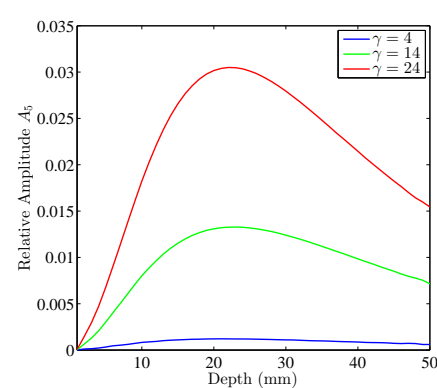

(c) At Second odd harmonic $5 f$

Figure 17. FEM simulations with different elastic parameters $\gamma$ : Evolution of the fundamental and odd harmonics components along the propagation distance. Note: circle marks in the Fig.(b) are the analytical results by Eq.23.

\subsection{Effect of the second non-linear elastic parameter $\zeta$}

Finally, different combinations of $A$ and $D$ are employed but the non-linear elastic coefficient $\gamma$ is kept the same. The materials are summarized in Tab.IV, it can be seen that, even if $\gamma$ is the same, varying the combinations between $A$ and $D$ still leads the second non-linear coefficient $\zeta\left(\zeta=\frac{A}{4}+\frac{3}{2} D\right.$, see Eq.32) to be different.

In the results, Fig.18(a) shows the same dissipation behaviour at the fundamental frequency for the three considered materials. For the analytical model in which $\zeta$ is not involved, the three materials lead to the same Goldberg number which equals 13.57, so only 
one analytical result is shown in Fig.18(b). In this figure, the three simulation results overlap with no much difference, but they are all slightly different from the analytical solution.

Then, for the second odd harmonic $5 f$ in Fig.(c), the difference among the three materials can be observed at the maximal amplitude. The gap is attributed to the difference of the second non-linear coefficient $\zeta$. For instance, $\zeta$ in material No.5 is the most important, so it has the largest harmonic amplitude (green curve). However, at the typical level of strain of elastography, this effect is too small to be accurately estimated with a plane wave approach, i.e., the maximal difference is only $0.2 \%$ compared to the load amplitude. So the second non-linear elastic parameter $\zeta$ is difficult to determine only by plane wave experiments.

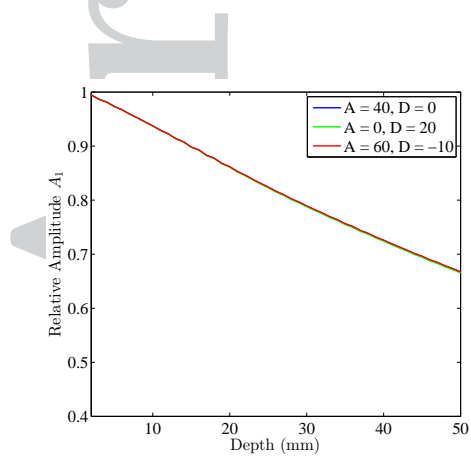

(a) At fundamental frequency $f$

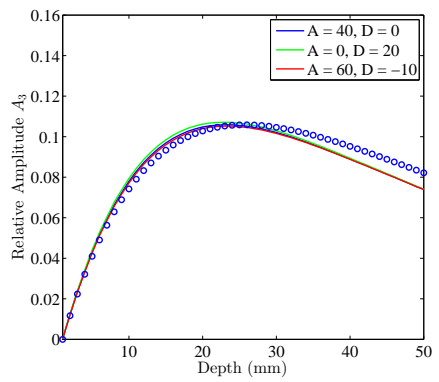

(b) At first odd harmonic $3 f$

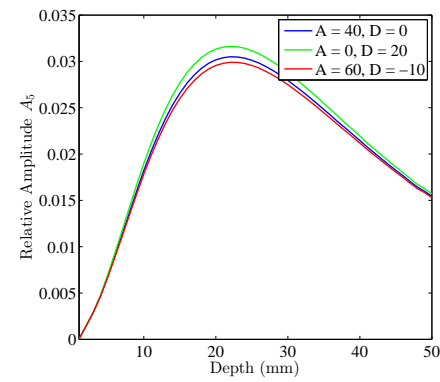

(c) At Second odd harmonic $5 f$

Figure 18. FEM simulations with different elastic parameters $\zeta$ : Evolution of the fundamental and odd harmonics components along the propagation distance. Note: the analytical results are the same for three cases, presented by blue circle marks in Fig.(b).

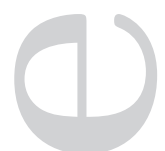

4.7. Discussion

In this section, the generation of odd harmonics of non-linear plane shear wave is studied with different parameters. The simulation results are compared to the existing analytical model, a good agreement is achieved at small Goldberg numbers. The comparison indicates that the analytical solution is accurate when the Goldberg number is smaller than about 10.

The simulations results are now displayed by the maximal amplitudes as a function of Goldberg number $\Gamma$, see Fig.19. For the first odd harmonic at $3 f$ (Fig.19(a)), the amplitude does not increase linearly with the Goldberg number, thus a quadratic term is involved in the fitting. Meanwhile, the second harmonic at $5 f$ (Fig.19(b)) shows a linear growth with the Goldberg number.

Finally, two equations respectively for the first and second harmonics are fitted in Eq.26. The fitting curves are also presented in Fig.19, a good agreement can be observed. 


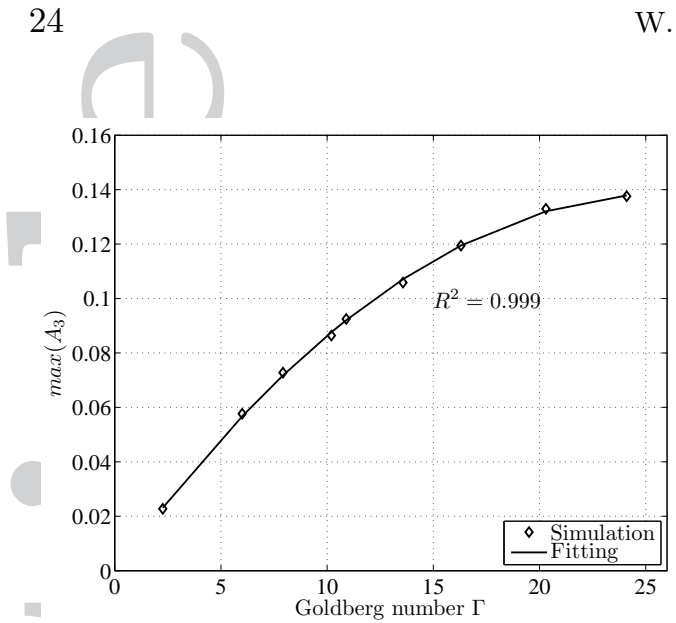

(a) The first odd harmonic: $A_{3}$

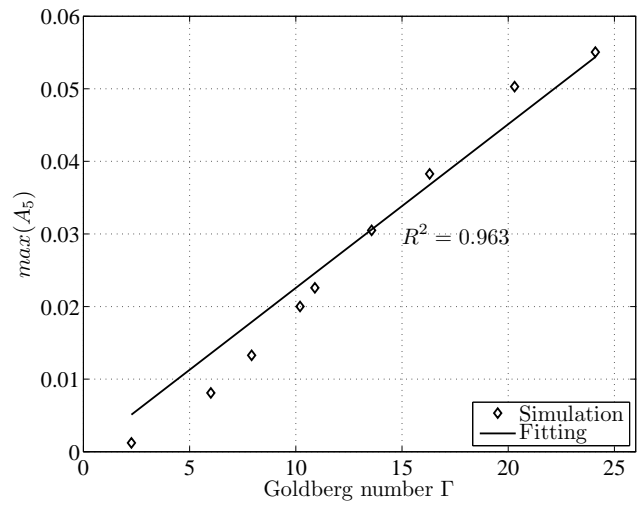

(b) The second odd harmonic: $A_{5}$

Figure 19. Fit of the relationship between the odd harmonics amplitude and Goldberg number

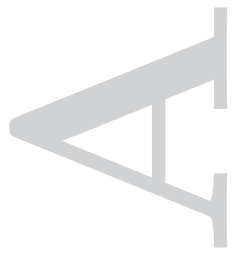

$$
\begin{aligned}
& \max \left(A_{3}\right)=-0.206 e^{-3} \times \Gamma^{2}+10.7 e^{-3} \times \Gamma \\
& \max \left(A_{5}\right)=2.26 e^{-3} \times \Gamma
\end{aligned}
$$

These relationships indicate that, the Goldberg number $\Gamma$ can be deduced for a homogeneous soft solid by measuring the harmonic components. In real life condition, plane wave in biological tissues can easily be generated by the Supersonic Shear Imaging (SSI) technique [3]. The linear parameters such as the shear modulus $\mu$ and the coefficient of viscosity $\eta$ are not difficult to determine [37]. Therefore, the non-linear coefficient $\gamma$ can be deduced by Eq.24.

Theoretically, both relations in Eq.26 can be used to determine the non-linear parameter $\gamma$, but the second odd harmonic amplitude is too small so that the application in real life would lead to large errors. So the equation of the first odd harmonic should be considered firstly.

At last, these relationships are valid for a large domain (Goldberg number $\Gamma$ from 0 to 25) which extends the analytical model to highly non-linear response. However, as presented in Section 4.6, harmonic measurement is not able to distinguish the two non-linear parameters $A$ and $D$. In order to achieve this objective, a complementary experiment is necessary. For example, one may measure the shear wave speed in different pre-stressed states [9]. 


\section{CONCLUSION}

In this work, nearly incompressible hyperelastic Landau's law is introduced into a FE formulation. It is compared to classical laws in the field of continuum mechanics, the model is close to Rivlin model in a range of limited deformation. However, neo-Hookean (and Mooney-Rivlin, see Appendix) model has an exactly linear behaviour in shear, so it can not be used to model non-linear shear waves.

The viscosity of soft solids is also considered for dynamic applications. Voigt model is employed because it describes accurately the viscoelastic behaviour of soft solids at low frequency. Numerical simulations show a good agreement with the experiments in the literature. However, it is necessary to point out that Voigt model is not considered to be validated at high frequency range.

In the parametric study, we propose a simple, forward method to identify the non-linear parameter of homogeneous soft solids. This is achieved by measuring the amplitudes of the first odd harmonics of non-linear plane waves. However, Landau's law contains two nonlinear parameters $A$ and $D$, and only the sum of them can be deduced by this method. In order to fully characterize Landau's material, i.e., identify $A$ and $D$ separately, another experiment should be involved, this is work in progress.

The visco-hyperelastic Landau's model has a broad perspective in numerical simulations of complex non-linear wave phenomena, such as real geometry, heterogeneity [43], diffraction [44] and focalisation [21] effect.

Moreover, Landau's FE model is potentially valuable for inverse problems. For example, characterizing tumours surrounded by healthy tissues is an important issue and it is often considered as an inverse problem. In the last decade, substantial progress has been made in linear elasticity $[4,5]$ and viscosity [45]. Further effort is necessary in the non-linear domain.

\section{ACKNOWLEDGEMENT}

The authors wish to thank the support of the CIFRE convention $N^{\circ} 2013-1553$.

\section{A. APPENDIX: ANALYTICAL EXPRESSION IN SHEAR}

In this appendix, we give the analytic expressions of Cauchy shear stress for the shear deformation described by Eq.18. These expressions are directly related to the generation of harmonics in nonlinear shear wave propagation presented in Section 3 and 4.

This article is protected by copyright. All rights reserved. 
Neo-Hookean and Mooney-Rivlin models have a purely linear relationship, they can be written as

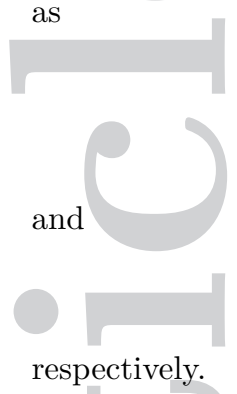

$$
\begin{gathered}
\sigma_{23}=2 c_{10} \kappa \\
\sigma_{23}=2\left(c_{10}+c_{01}\right) \kappa
\end{gathered}
$$

For Rivlin model which includes a higher order term, we have:

$$
\sigma_{23}=2\left(c_{10}+c_{01}\right) \kappa+4 c_{20} \kappa^{3}
$$

And for Landau's material, the shear stress is expressed by:

$$
\sigma_{23}=\mu \kappa+\left(\mu+\frac{A}{2}+D\right) \kappa^{3}+\left(\frac{A}{4}+\frac{3}{2} D\right) \kappa^{5}+D \kappa^{7}
$$

In Eq.30, the last two terms have slight effect at the level of strain of non-linear shear waves. In a number of work $[11,12,14,1]$, these terms are neglected, so Landau's model clearly coincides with Rivlin model, and the relationships in Eq.16 is recovered. Besides, the coefficient at the cubic term is defined as the non-linear coefficient in plane shear wave $\gamma$ :

$$
\gamma=\mu+\frac{A}{2}+D
$$

In this work, we define the second non-linear coefficient $\zeta$ which is the coefficient at the fifth term in Eq.30, so

$$
\zeta=\frac{A}{4}+\frac{3}{2} D
$$

This coefficient has very small effect at the typical level of wave propagation, its utility is discussed in Section 4.

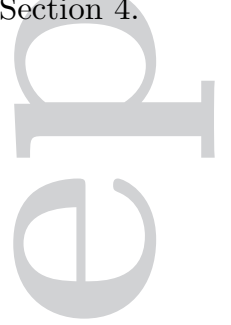

\section{REFERENCES}

1. Jacob X, Catheline S, Gennisson JL, Barrière C, Royer D, Fink M. Nonlinear shear wave interaction in soft solids. The Journal of the Acoustical Society of America 2007; 122(4):1917-1926.

2. Catheline S, Wu F, Fink M. A solution to diffraction biases in sonoelasticity: the acoustic impulse technique. The Journal of the Acoustical Society of America 1999; 105(5):2941-2950.

3. Bercoff J, Tanter M, Fink M. Supersonic shear imaging: a new technique for soft tissue elasticity mapping. Ultrasonics, Ferroelectrics and Frequency Control, IEEE Transactions on 2004; 51(4):396409.

4. McLaughlin J, Renzi D. Shear wave speed recovery in transient elastography and supersonic imaging using propagating fronts. Inverse Problems 2006; 22(2):681.

This article is protected by copyright. All rights reserved. 
5. McLaughlin J, Renzi D. Using level set based inversion of arrival times to recover shear wave speed in transient elastography and supersonic imaging. Inverse Problems 2006; 22(2):707.

6. Catheline S, Gennisson JL, Tanter M, Fink M. Observation of shock transverse waves in elastic media. Physical review letters 2003; 91(16):164301.

7. Gennisson JL, Rénier M, Catheline S, Barrière C, Bercoff J, Tanter M, Fink M. Acoustoelasticity in soft solids: Assessment of the nonlinear shear modulus with the acoustic radiation force. The Journal of the Acoustical Society of America 2007; 122(6):3211-3219.

8. Catheline S, Gennisson JL, Fink M. Measurement of elastic nonlinearity of soft solid with transient elastography. The Journal of the Acoustical Society of America 2003; 114(6):3087-3091.

9. Jiang Y, Li G, Qian LX, Liang S, Destrade M, Cao Y. Measuring the linear and nonlinear elastic properties of brain tissue with shear waves and inverse analysis. Biomechanics and modeling in mechanobiology 2015; 14(5):1119-1128.

10. Jiang Y, Li GY, Qian LX, Hu XD, Liu D, Liang S, Cao Y. Characterization of the nonlinear elastic properties of soft tissues using the supersonic shear imaging (ssi) technique: inverse method, ex vivo and in vivo experiments. Medical image analysis 2015; 20(1):97-111.

11. Zabolotskaya EA, Hamilton MF, Ilinskii YA, Meegan GD. Modeling of nonlinear shear waves in soft solids. The Journal of the Acoustical Society of America 2004; 116(5):2807-2813.

12. Wochner MS, Hamilton MF, Ilinskii YA, Zabolotskaya EA. Cubic nonlinearity in shear wave beams with different polarizations. The Journal of the Acoustical Society of America 2008; 123(5):2488-2495.

13. Destrade M, Gilchrist MD, Saccomandi G. Third-and fourth-order constants of incompressible soft solids and the acousto-elastic effect. The Journal of the Acoustical Society of America 2010; 127(5):2759-2763.

14. Michel Destrade RWO. On the third- and fourth-order constants of incompressible isotropic elasticity. The Journal of the Acoustical Society of America 2010; .

15. Chen Q, Ringleb SI, Manduca A, Ehman RL, An KN. A finite element model for analyzing shear wave propagation observed in magnetic resonance elastography. Journal of Biomechanics 2005; 38(11):2198 -2203 .

16. Chen Q, Ringleb SI, Manduca A, Ehman RL, An KN. Differential effects of pre-tension on shear wave propagation in elastic media with different boundary conditions as measured by magnetic resonance elastography and finite element modeling. Journal of biomechanics 2006; 39(8):1428-1434.

17. Palmeri ML, Sharma AC, Bouchard RR, Nightingale RW, Nightingale KR. A finite-element method model of soft tissue response to impulsive acoustic radiation force. Ultrasonics, Ferroelectrics and Frequency Control, IEEE Transactions on 2005; 52(10):1699-1712.

18. Lee KH, Szajewski BA, Hah Z, Parker KJ, Maniatty AM. Modeling shear waves through a viscoelastic medium induced by acoustic radiation force. International journal for numerical methods in biomedical engineering 2012; 28(6-7):678-696.

19. Park 1 E, Maniatty AM. Finite element formulation for shear modulus reconstruction in transient elastography. Inverse Problems in Science and Engineering 2009; 17(5):605-626.

20. Zhao X, Pelegri AA. A bayesian approach for characterization of soft tissue viscoelasticity in acoustic radiation force imaging. International journal for numerical methods in biomedical engineering 2015; .

21. Giammarinaro B, Coulouvrat F, Pinton G. Numerical simulation of focused shock shear waves in soft solids and a two-dimensional nonlinear homogeneous model of the brain. Journal of biomechanical engineering 2016; 138(4):041 003.

22. Khelladi H. Goldberg's Number Influence on the Validity Domain of the Quasi-Linear Approximation of Finite Amplitude Acoustic Waves. INTECH Open Access Publisher, 2012.

23. Saenger EH, Bohlen T. Finite-difference modeling of viscoelastic and anisotropic wave propagation using the rotated staggered grid. Geophysics 2004; 69(2):583-591.

This article is protected by copyright. All rights reserved. 
24. Alshaikh IA, Turhan D, Mengi Y. Two-dimensional transient wave propagation in viscoelastic layered media. Journal of Sound and vibration 2001; 244(5):837-858.

25. Moczo P, Kristek J. On the rheological models used for time-domain methods of seismic wave propagation. Geophysical Research Letters 2005; 32(1).

26. Holzapfel GA. Nonlinear solid mechanics, vol. 24. Wiley Chichester, 2000.

27. Rivlin R. Large elastic deformations of isotropic materials. iv. further developments of the general theory. Philosophical Transactions of the Royal Society of London A: Mathematical, Physical and Engineering Sciences 1948; 241(835):379-397.

28. Mangan R, Destrade M, Saccomandi G. Strain energy function for isotropic non-linear elastic incompressible solids with linear finite strain response in shear and torsion. Extreme Mechanics Letters 2016; :-

29. Destrade M, Gilchrist MD, Murphy JG. Onset of nonlinearity in the elastic bending of blocks. Journal of Applied Mechanics 2010; 77(6):061 015.

30. Landau LD, Lifshitz EM. Theory of Elasiticy. Pergamon, New York, 1986.

31. Bonet J, Wood RD. Nonlinear continuum mechanics for finite element analysis. Cambridge university press, 1997.

32. Hamilton MF, Ilinskii YA, Zabolotskaya EA. Separation of compressibility and shear deformation in the elastic energy density (1). Acoustical Society of America Journal 2004; 116:41-44.

33. Hibbitt K, Sorensen. ABAQUS/CAE User's Manual. Hibbitt, Karlsson \& Sorensen, Incorporated, 2002.

34. Rénier M, Gennisson JL, Barrière C, Royer D, Fink M. Fourth-order shear elastic constant assessment in quasi-incompressible soft solids. Appl. Phys. Lett 2008; 93(10):101 912-101912.

35. Sussman T, Bathe KJ. A model of incompressible isotropic hyperelastic material behavior using spline interpolations of tension-compression test data. International Journal for Numerical Methods in Biomedical Engineering 2009; 25(1):53-63.

36. Ogden RW. Non-linear elastic deformations. Courier Corporation, 1997.

37. Catheline S, Gennisson JL, Delon G, Fink M, Sinkus R, Abouelkaram S, Culioli J. Measurement of viscoelastic properties of homogeneous soft solid using transient elastography: an inverse problem approach. The Journal of the Acoustical Society of America 2004; 116(6):3734-3741.

38. Bower AF. Applied mechanics of solids. CRC press, 2011.

39. Belytschko T, Liu WK, Moran B, Elkhodary K. Nonlinear finite elements for continua and structures. John Wiley \& Sons, 2013.

40. Destrade M, Saccomandi G, Vianello M. Proper formulation of viscous dissipation for nonlinear waves in solids. The Journal of the Acoustical Society of America 2013; 133(3):1255-1259.

41. Ye W, Bel-Brunon A, Catheline S, Rochette M, Combescure A. A selective mass scaling method for shear wave propagation analyses in nearly incompressible materials. International Journal for Numerical Methods in Engineering 2016; .

42. Catheline S, Benech N. Longitudinal shear wave and transverse dilatational wave in solids. The Journal of the Acoustical Society of America 2015; 137(2):EL200-EL205.

43. Sandrin L, Tanter M, Catheline S, Fink M. Shear modulus imaging with 2-d transient elastography. Ultrasonics, Ferroelectrics and Frequency Control, IEEE Transactions on 2002; 49(4):426-435.

44. Catheline S, Thomas JL, Wu F, Fink MA. Diffraction field of a low frequency vibrator in soft tissues using transient elastography. IEEE transactions on ultrasonics, ferroelectrics, and frequency control 1999; 46(4):1013-1019.

45. McLaughlin J, Thomas A, Yoon JR. Basic theory for generalized linear solid viscoelastic models. AMS Contemporary Mathematics Volume: Tomography and Inverse Transport Theory, edited by G. Bal, D. Finch, P. Kuchment, J. Schotland, P. Stefanov, and G. Uhlmann. Providence: American Mathematical 
Society $2011 ;: 101-134$.
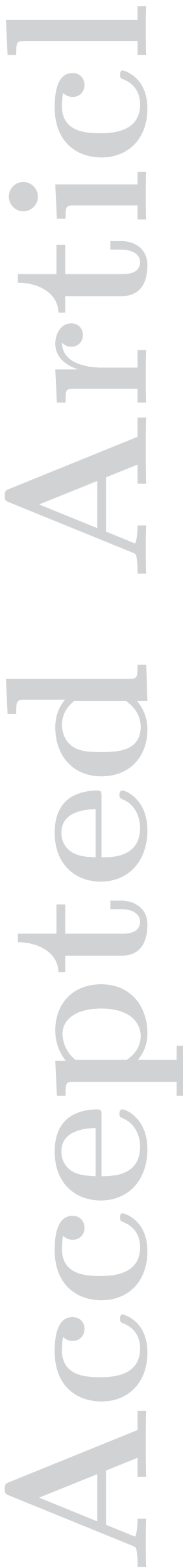

This article is protected by copyright. All rights reserved. 